\title{
SPSB1-mediated HnRNP A1 ubiquitylation regulates alternative splicing and cell migration in EGF signaling
}

Feng Wang ${ }^{1}$, Xing $\mathrm{Fu}^{2}$, Peng $\mathrm{Chen}^{3}$, Ping $\mathrm{Wu}^{4,5}$, Xiaojuan $\mathrm{Fan}^{6}{ }^{6} \mathrm{Na} \mathrm{Li}^{1}$, Hong Zhu ${ }^{1}$, Ting-Ting $\mathrm{Jia}^{1}$, Hongbin $\mathrm{Ji}^{3}$, Zefeng Wang ${ }^{6}$, Catherine C L Wong, ${ }^{4,5}$, Ronggui $\mathrm{Hu}^{3}$, Jingyi Hui ${ }^{1}$

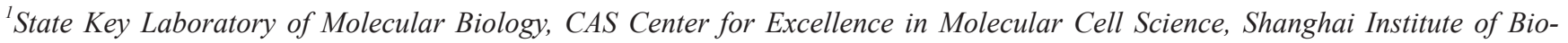
chemistry and Cell Biology, Chinese Academy of Sciences; University of Chinese Academy of Sciences, Shanghai 200031, China; ${ }^{2}$ Shanghai Center for Plant Stress Biology, Chinese Academy of Sciences, Shanghai 201602, China; ${ }^{3}$ Key Laboratory of Systems Biology, CAS Center for Excellence in Molecular Science, Innovation Center for Cell Signaling Network, Shanghai Institute of Biochemistry and Cell Biology, Chinese Academy of Sciences, Shanghai 200031, China; ${ }^{4}$ National Center for Protein Science Shanghai, Shanghai Institute of Biochemistry and Cell Biology, Chinese Academy of Sciences, Shanghai 200031, China, ${ }^{5}$ Shanghai Science Research Center, Chinese Academy of Sciences, Shanghai 201204, China; ${ }^{6}$ Key Lab of Computational Biology, CAS Center for Excellence in Molecular Cell Science, CAS-MPG Partner Institute for Computational Biology, Chinese Academy of Sciences, Shanghai 200031, China

Extracellular signals have been shown to impact on alternative pre-mRNA splicing; however, the molecular mechanisms and biological significance of signal-induced splicing regulation remain largely unknown. Here, we report that epidermal growth factor (EGF) induces splicing changes through ubiquitylation of a well-known splicing regulator, hnRNP A1. EGF signaling upregulates an E3 ubiquitin (Ub) ligase adaptor, SPRY domain-containing SOCS box protein 1 (SPSB1), which recruits Elongin B/C-Cullin complexes to conjugate lysine 29-linked polyUb chains onto hnRNP A1. Importantly, SPSB1 and ubiquitylation of hnRNP A1 have a critical role in EGF-driven cell migration. Mechanistically, EGF-induced ubiquitylation of hnRNP A1 together with the activation of SR protein kinases (SRPKs) results in the upregulation of a Rac1 splicing isoform, Rac1b, to promote cell motility. These findings unravel a novel crosstalk between protein ubiquitylation and alternative splicing in EGF/EGF receptor signaling, and identify a new EGF/SPSB1/hnRNP A1/Rac1 axis in modulating cell migration, which may have important implications for cancer treatment.

Keywords: alternative splicing; EGF signaling; hnRNP A1; protein ubiquitylation; Rac1; SPSB1

Cell Research (2017) 27:540-558. doi:10.1038/cr.2017.7; published online 13 January 2017

\section{Introduction}

Alternative pre-mRNA splicing is a widespread mechanism that contributes to the expansion of genome complexity and the control of gene expression at the post-transcriptional level $[1,2]$. Through alternative splicing, multiple transcripts are generated from a single gene, leading to the production of functionally different or even opposing protein isoforms. Alternative splicing

Correspondence: Jingyi Hui ${ }^{\mathrm{a}}$, Ronggui $\mathrm{Hu}^{\mathrm{b}}$

${ }^{a}$ E-mail: jyhui@sibcb.ac.cn

bE-mail: coryhu@sibcb.ac.cn

Received 28 November 2016; revised 6 December 2016; accepted 9 December 2016; published online 13 January 2017 also occurs in the $5^{\prime}$-untranslated and $3^{\prime}$-untranslated regions, which can affect mRNA stability and translation. Current models propose that trans-acting factors, most of them are RNA-binding proteins, recognize the cis-elements localized in the pre-mRNAs and determine splice sites by modulating spliceosome assembly [3-5].

Signal transduction is an important mechanism for cells to respond to extracellular changes and maintain homeostasis. Dysregulation of signal transduction pathways has been heavily implicated in human diseases including a variety of cancers. At the molecular level, much effort has been made to investigate how signal transduction cascades sense, transmit and amplify signals to regulate cellular processes by inducing gene expression changes at the transcriptional and translational levels. Fewer 
studies have focused on understanding how extracellular signals are linked to gene expression regulation at the post-transcriptional level .

It is known that alternative splicing is subject to the control of various extracellular stimuli [6-8]. For example, using high-throughput methods, substantial splicing changes have been detected during $\mathrm{T}$ cell activation or after the stimulation of insulin, wingless or epidermal growth factor (EGF) [9-12]. During T cell activation, it was reported recently that JNK signaling constitutes a major signal transduction pathway for regulated splicing and induces a positive feedback loop through CELF2-mediated control of MKK7 splicing [13]. In EGF signaling, Akt activation appears to have a major role in regulating alternative splicing through Akt-induced autophosphorylation, nuclear translocation of SR protein kinases (SRPKs) and phosphorylation of SR proteins by SRPKs [11]. However, so far, our understanding of the mechanisms underlying signal-induced changes in splicing program remains fragmentary and incomplete. Furthermore, little is known about the functional significance of regulated splicing in signal transduction.

EGF signaling is essential for a variety of cellular processes including cell growth and migration [14, 15]. EGF-driven cellular responses involve EGF binding with EGF receptor (EGFR, an ErbB family receptor tyrosine kinase), dimerization and phosphorylation of EGFR, and subsequent initiation of a vast array of signal transduction pathways. Aberrant activation of EGF/EGFR signaling has key roles in the pathogenesis and progression of human cancers.

To understand the regulation and consequences of EGF-induced alternative splicing, we systematically assessed EGF-induced splicing changes using RNA-seq, and dissected the regulatory cis-elements that respond to EGF signaling. We found an enrichment of hnRNP A1-binding motif in EGF-activated exons, which led us to uncover that hnRNP A1 is modified by ubiquitylation upon EGF stimulation. Further studies demonstrated that SPRY domain-containing SOCS box protein 1 (SPSB1)-mediated ubiquitylation of hnRNP A1 is involved in regulating alternative splicing and cell migration in EGF/ EGFR signaling.

\section{Results}

\section{HnRNP A1 is ubiquitylated upon EGF treatment}

To understand the regulation and biological significance of EGF-induced alternative splicing, we performed a high-throughput transcriptomic analysis to assess splicing changes upon EGF stimulation in HeLa cells (a model system for investigation of EGF signaling).
Total RNAs were isolated from HeLa cells $4 \mathrm{~h}$ after control or EGF treatment followed by polyA ${ }^{+}$RNA sequencing. We detected 416 altered cassette exon events (Benjamini-Hochberg adjusted $p$-value $<0.05$, splicing change $>0.15$, Supplementary information, Table S1). Using alternative exon- and exon-exon junction-specific primers, we validated a subset of splicing events in Supplementary information, Table S1 by real-time RT-qPCR (Supplementary information, Figure S1). To characterize the cis-elements that respond to EGF stimulation, we analyzed sequence motifs enriched within the alternative exons and in the flanking intronic regions (containing the first and last $300 \mathrm{nt}$ of the flanking introns, excluding the first 9 and last $30 \mathrm{nt}$ of the intron sequences which contain the $5^{\prime}$ and $3^{\prime}$ splice sites, respectively). Through comparing the enriched motifs with the binding sites for RNA-binding proteins characterized previously [16], we found that the RNA-binding motif UAGGG for hnRNP A1 protein is significantly enriched in EGF-activated cassette exons (Figure 1A, Supplementary information, Table S2), suggesting that hnRNP A1 might have a prominent role in mediating EGF-induced alternative splicing. HnRNP A1, one of the ubiquitously expressed heterogeneous nuclear ribonucleoproteins ( $h n R N P s$ ), is known to function in both constitutive and alternative splicing [1720]. To examine the role of hnRNP A1 in EGF-induced splicing regulation, we assessed splicing changes upon knockdown of hnRNP A1 in HeLa cells using RNA-seq and compared them with EGF-induced splicing changes. In total, among 15295 cassette exon-type events detected in both EGF treatment and hnRNP A1 knockdown cells, we identified 646 and 918 events with significant changes after EGF treatment and hnRNP A1 knockdown, respectively (Benjamini-Hochberg adjusted $p$-value $<$ 0.05 , splicing change $>0.10$ ). The overlapping events between these two groups are 158 cases (odds ratio $=$ 5.92, $p$-value $<4.2 \mathrm{e}-20$, Figure 1B, Supplementary information, Table S3). Interestingly, splicing changes induced by EGF treatment have a significantly positive correlation with those caused by hnRNP A1 knockdown $(R=0.84$, Figure $1 \mathrm{C})$. These results strongly suggest that reduced expression or functionality of hnRNP A1 may account for a significant portion of EGF-induced splicing events.

Since the expression level of hnRNP A 1 protein showed no significant change during the time course of EGF treatment (Figure 1D), it is likely that EGF signaling may activate a post-translational mechanism to regulate hnRNP A1 function. Intriguingly, although the phosphorylation or acetylation status of hnRNP A1 remained unchanged after EGF treatment (data not shown), ubiquitylation of hnRNP A1 was stimulated dramatically 
A

282 up-regulated cassette exons

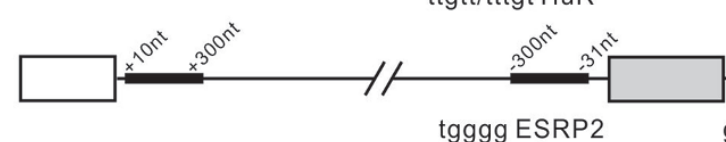

\section{4 down-regulated} cassette exons

B

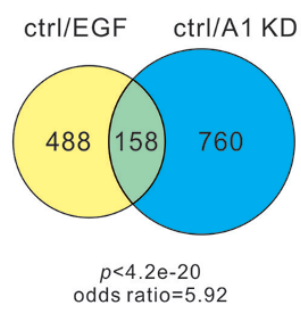

D

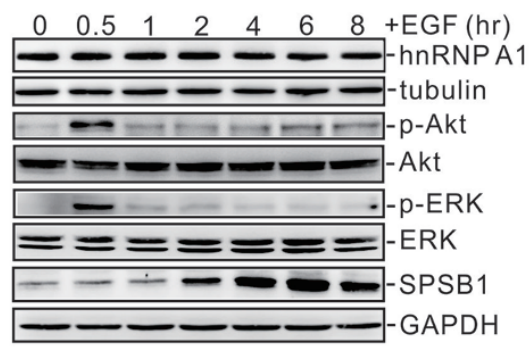

$\mathbf{F}$
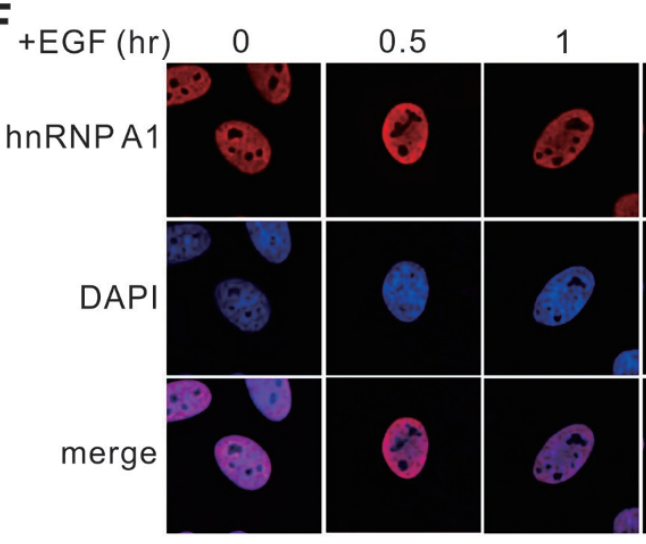

taggg (hnRNP A1)

taaaa/ataaa KHDRBS1/2/3 aaaa SART3/PABP tttgt/ttgtt HuR taatt A1CF cttaa ZCRB1

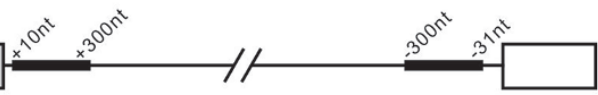

gacga ANKHD1/RBM45

$p<0.001$

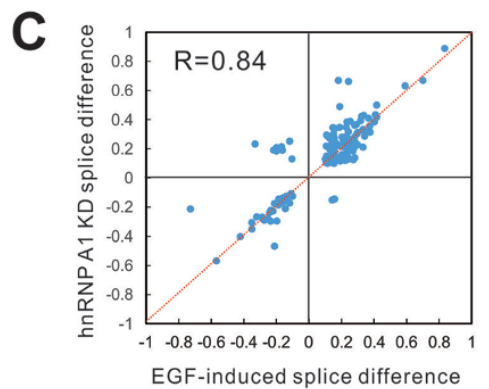

E

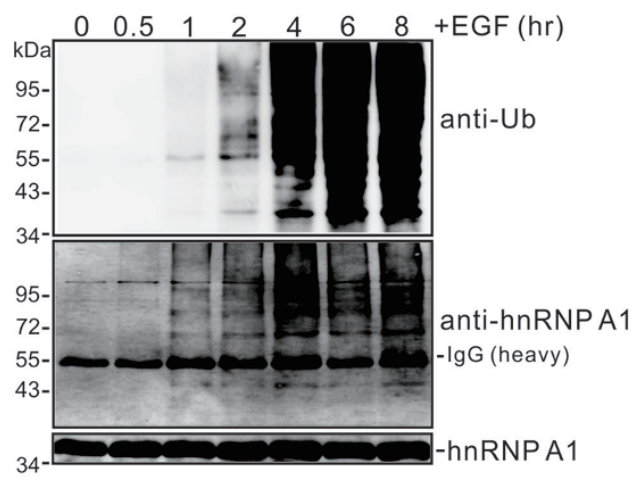

\section{2}
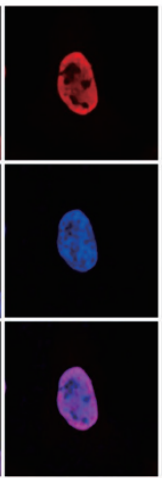

4
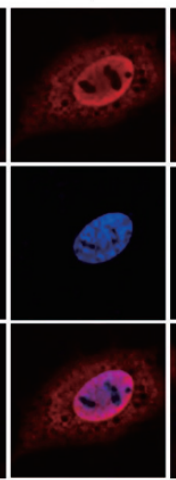

6

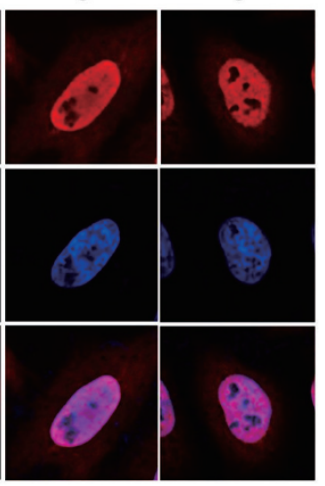

Figure 1 EGF induces the ubiquitylation of hnRNP A1. (A) Motif enrichment analysis of EGF-responding cis-elements in the cassette exons (grey box) and its flanking intronic regions (Benjamini-Hochberg adjusted $p$-value $<0.05$, splicing change $>$ 0.15). Black boxes contain the first and last $300 \mathrm{nt}$ of the flanking introns, excluding the first 9 and last $30 \mathrm{nt}$ of the intron sequences. (B) Venn chart depicting the overlap of cassette exon-type splicing changes (Benjamini-Hochberg adjusted $p$-value $<0.05$, splicing change $>0.10$ ) induced by EGF treatment and upon hnRNP A1 knockdown. (C) Scatter plot showing the comparison between splicing changes induced by EGF treatment and those upon hnRNP A1 knockdown. The $Y$ and $X$ axes represent changes in exon inclusion ratios. (D) Immunoblotting analysis of the expression levels of hnRNP A1, p-Akt, total Akt, p-ERK, total ERK and SPSB1 in HeLa cells treated with EGF for the time indicated. (E) Ubiquitylation status of endogenous hnRNP A1 in HeLa cells treated with EGF. Immunoprecipitated endogenous hnRNP A1 with a monoclonal antibody from cell extracts collected at the time indicated after EGF treatment was immunoblotted with an anti-Ub antibody (upper panel) or a polyclonal anti-hnRNP A1 antibody (lower panel). The immunoprecipitates loaded onto the gels were normalized at the level of hnRNP A1. (F) Immunostaining of hnRNP A1 in HeLa cells treated with EGF for the time indicated. 
$4 \mathrm{~h}$ after EGF was added (Figure 1E). Modification of hnRNP A1 by ubiquitin (Ub) was also detected in A549 lung adenocarcinoma cells and EpH4 mammary epithelial cells after EGF treatment (Supplementary information, Figure S2A and S2B). In addition, we observed that a fraction of hnRNP A1 was localized to the cytoplasm starting from $4 \mathrm{~h}$ after EGF stimulation in HeLa cells (Figure 1F).

SPSB1 interacts with hnRNP Al and is required for ubiquitylation of hnRNP A1 in EGF/EGFR signaling

To understand the function and mechanism of hnRNP A1 ubiquitylation in EGF signaling, we set out to identify the E3 Ub ligase for hnRNP A1 through a yeast two-hybrid (Y2H) screening. HnRNP A1 was used as the bait to screen potential hnRNP A1-interacting proteins from a cDNA library encoding over 400 E3 Ub ligases or their substrate-binding subunits as described previously [21]. In total, we obtained nine positive colonies, of which seven colonies contained open reading frame (ORF) for SPSB1 (SPRY (sp1A/ryanodine receptor) domain-containing SOCS (suppressor of cytokine signaling) box protein 1; Figure 2A), whereas the other two colonies for RBCK1 (RANBP2-type and C3HC4-type zinc finger containing 1). Since knockdown of RBCK1 in HeLa cells did not affect EGF-induced hnRNP A1 ubiquitylation (data not shown), we focused on SPSB1 in the rest of this study. To validate the potential interaction between SPSB1 and hnRNP A1, we performed immunoprecipitation, co-immunoprecipitation and in vitro GST pulldown assays, and found that either endogenous or ectopically expressed hnRNP A1 interacted with SPSB1 in a DNA- and RNA-independent manner (Figure 2B and 2C), and that the recombinant GST-SPSB1 and His-hnRNP A1 proteins purified from bacteria directly bound to each other (Figure 2D). Using a series of hnRNP A1 truncation or deletion constructs, the SPSB1-interacting domain in hnRNP A1 was mapped to its C-terminal 15 amino acid residues (Figure 2E and 2F).

SPSB1, originally identified through searching for SOCS box-containing proteins, is one member of the SPRY domain-containing SOCS box (SSB) protein family [22]. Members of this family and other SOCS box-containing proteins have been reported to act as adaptors to bring the SOCS box-associated E3 Ub ligase complexes to their substrates for ubiquitylation and proteasomal degradation [23]. Interestingly, we noticed that SPSB1 was upregulated upon EGF stimulation in HeLa, A549 and EpH4 cells (Figure 1D, Supplementary information, Figure S2C and S2D), and consistently, EGF treatment enhanced the binding between endogenous hnRNP A1 and SPSB1 (Figure 2B). To test whether SPSB1 induces the ubiquitylation of hnRNP A1, we examined the ubiquitylation status of the immuno-purified hnRNP A1 from cells expressing wild-type SPSB1 or a SPSB1 mutant lacking the SOCS box domain. Ectopically expressed wild-type SPSB1 but not the mutant promoted the ubiquitylation of hnRNP A1, indicating that the adaptor function of SPSB1 was indeed required for hnRNP A1 ubiquitylation (Figure 2G, lanes 1-3). Consistently, ectopically expressed SPSB1 did not lead to the ubiquitylation of an hnRNP A1 mutant missing the last 15 amino acids (Figure 2G, lanes 4-6).

To further determine whether SPSB1 is responsible for EGF-induced ubiquitylation of hnRNP A1, we treated control or SPSB1 knockdown cells with EGF. The $\mathrm{Ub}$ modification of hnRNP A1 did not respond to EGF upon knockdown of SPSB1 (Figure 2H). Importantly, the EGFR inhibitor Gefitinib blocked hnRNP A1 ubiquitylation and repressed the upregulation of SPSB1 expression (Figure 2I). These data indicate that EGF/EGFR signaling induces the ubiquitylation of hnRNP A1 through upregulation of SPSB1.

\section{SPSB1 recruits Elongin B/C-Cullin complexes to ubiqui-} tylate hnRNP Al both in vivo and in vitro

SOCS box-containing proteins have been shown to act as substrate-recognition modules in the Elongin B/ C-Cullin-SOCS box (ECS) family of E3 ligase complexes [22-26]. Elongin B/C-Cul2 and Elongin B/C-Cul5 E3 Ub ligase complexes are two representatives of ECS family. Through immunoprecipitation assay using anti-SPSB1 antibody, we found that endogenous SPSB1 associated with Elongin B, Elongin C, Cul2 and Cul5 in HeLa cells with or without EGF treatment (Figure 3A). To test whether these components of ECS E3 complexes mediate EGF-induced hnRNP A1 ubiquitylation, we depleted them in HeLa cells by transfecting specific siRNAs. Single knockdown of Elongin B, Elongin C, Cul2 or Cul5 did not affect EGF-induced hnRNP A1 ubiquitylation (data not shown). However, double knockdown of Elongin $\mathrm{B}$ and $\mathrm{C}$ or of $\mathrm{Cul} 2$ and $\mathrm{Cul} 5$ by co-transfecting two targeting siRNAs apparently inhibited EGF-induced hnRNP A1 ubiquitylation (Figure 3B). The remaining $\mathrm{Ub}$ signals could be due to incomplete knockdown or the presence of other SPSB1-interacting E3 ligase complexes. These results suggest that SPSB1 is able to recruit both Elongin $\mathrm{B} / \mathrm{C}-\mathrm{Cul} 2$ and Elongin $\mathrm{B} / \mathrm{C}-\mathrm{Cul} 5$ complexes to ubiquitylate hnRNP A1 in cells. To test this hypothesis directly, we purified the Elongin B/C-Cul5RBX2 complex via GST-SPSB1 fusion protein (Figure $3 \mathrm{C}$ ) and performed in vitro ubiquitylation assay. Notably, the recombinant GST-hnRNP A1 could be ubiquitylated by GST-SPSB1 protein via recruiting Elongin B/C-Cul5- 
A

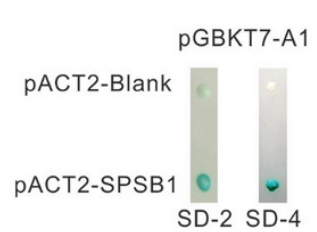

D

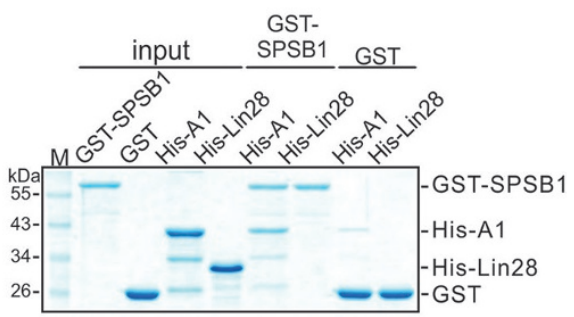

F

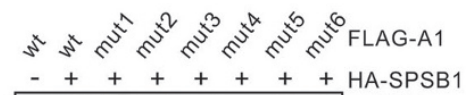

-+++++++ HA-SPSB
$+\infty-\infty-\infty$ anti-HA

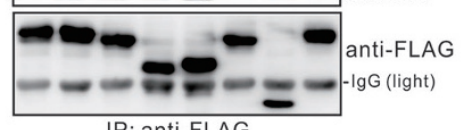

IP: anti-FLAG

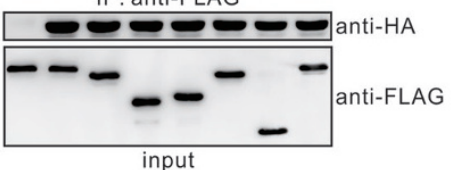

H

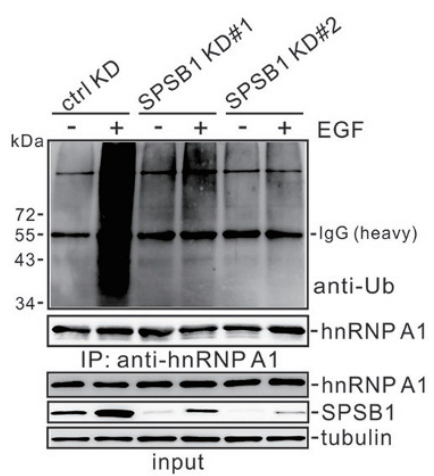

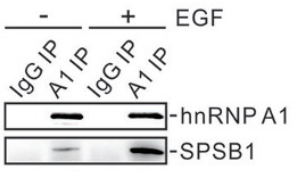

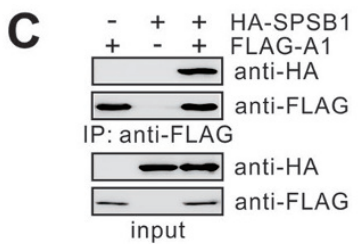

E

hnRNPA1

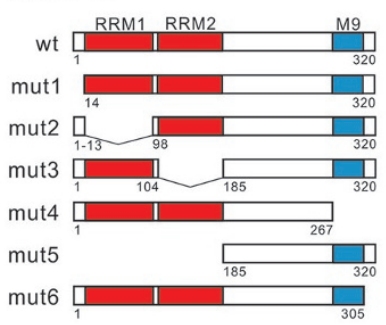

G

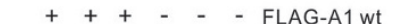

$+\quad+\quad+\quad-\quad-\quad-$ FLAG-A1 wt

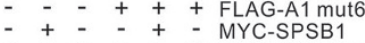

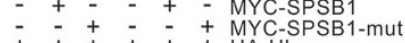
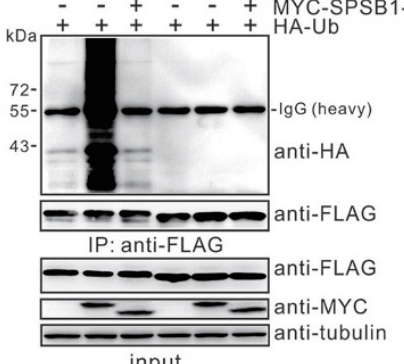

input

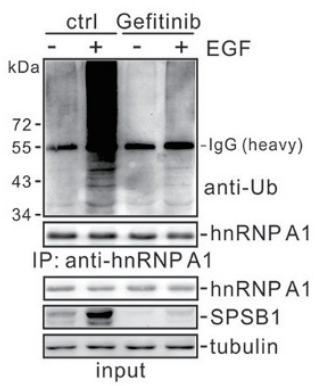

Figure 2 SPSB1 mediates hnRNP A1 ubiquitylation upon EGF/EGFR signaling. (A) SPSB1 was identified as an interacting protein of hnRNP A1 in a Y2H screen. SD-2, deficient in Leu and Trp; SD-4, deficient in Leu, Trp, His and Ura. (B) Endogenous SPSB1 interacts with hnRNP A1 in a DNA- and RNA-independent manner. Immunoprecipitation was performed with anti-hnRNP A1 antibody immobilized on Protein G Sepharose beads in the presence of DNase I and RNase A. The immunoprecipitates were detected by immunoblotting using anti-SPSB1 or anti-hnRNP A1 antibodies. (C) HA-tagged SPSB1 interacts with FLAG-tagged hnRNP A1. Immunoprecipitation was performed with anti-FLAG M2 beads in the presence of DNase I and RNase A. The immunoprecipitates were detected by immunoblotting using anti-FLAG or anti-HA antibodies. (D) SPSB1 binds hnRNP A1 directly in vitro. GST and GST-SPSB1 immobilized on Glutathione Sepharose were incubated with recombinant His-hnRNP A1 or His-Lin28 (as a control). After washing with buffer containing $300 \mathrm{mM} \mathrm{KCl}$, bound protein was separated by SDS-PAGE and visualized by Coomassie blue staining. (E) Schematic representation of wild type and mutant hnRNP A1. (F) Co-immunoprecipitation of FLAG-tagged hnRNP A1 and HA-tagged SPSB1 as in C. (G) SPSB1 ubiquitylates hnRNP A1. Immunoprecipitated FLAG-tagged wild-type hnRNP A1 or a hnRNP A1 mutant without the last 15 amino acid residues from HEK 293 cells expressing MYC-tagged wild-type SPSB1 or a SPSB1 mutant with SOCS box domain deleted was immunoblotted with anti-HA antibody. (H) SPSB1 is required for EGF-induced hnRNP A1 ubiquitylation. Immunoprecipitated endogenous hnRNP A1 from control or SPSB1 knockdown HeLa cells treated with or without EGF was immunoblotted with anti-Ub antibody. (I) EGFR inhibitor Gefitinib blocks EGF-induced hnRNP A1 ubiquitylation. Immunoprecipitated endogenous hnRNP A1 from HeLa cells with or without Gefitnib and EGF treatment was immunoblotted with anti-Ub antibody. 
RBX2 complex (Figure 3D). Thus, our data indicate that SPSB1 functions as an adaptor protein to mediate hnRNP A1 ubiquitylation by Elongin B/C-Cullin complexes.

SPSB 1 mediates the conjugation of K29-linked polyUb chains onto the Lys 183 and Lys 298 residues of hnRNP A1 mainly in the nucleus and induces cytoplasmic retention of a fraction of hnRNP Al

Using wild-type $\mathrm{Ub}$ and $\mathrm{Ub}$ mutants carrying a single lysine at residue $6,11,27,29,33,48$ or 63 (K6,
K11, K27, K29, K33, K48 or K63) or lysine-to-arginine substitutions on all lysine residues (K0), we found that SPSB1 preferentially conjugated K29-linked polyUb chains onto hnRNP A1 (Figure 4A). HnRNP A1 contains 18 lysine residues (Supplementary information, Figure S3A). To identify the lysines on the hnRNP A1 that are linked to $\mathrm{Ub}$, domain deletion and point mutation analyses were carried out. Removal of the whole RNA recognition motif 2 (RRM2) and the amino acid residues between 161 and 185 in RRM2 reduced hnRNP A1 ubiq-
A
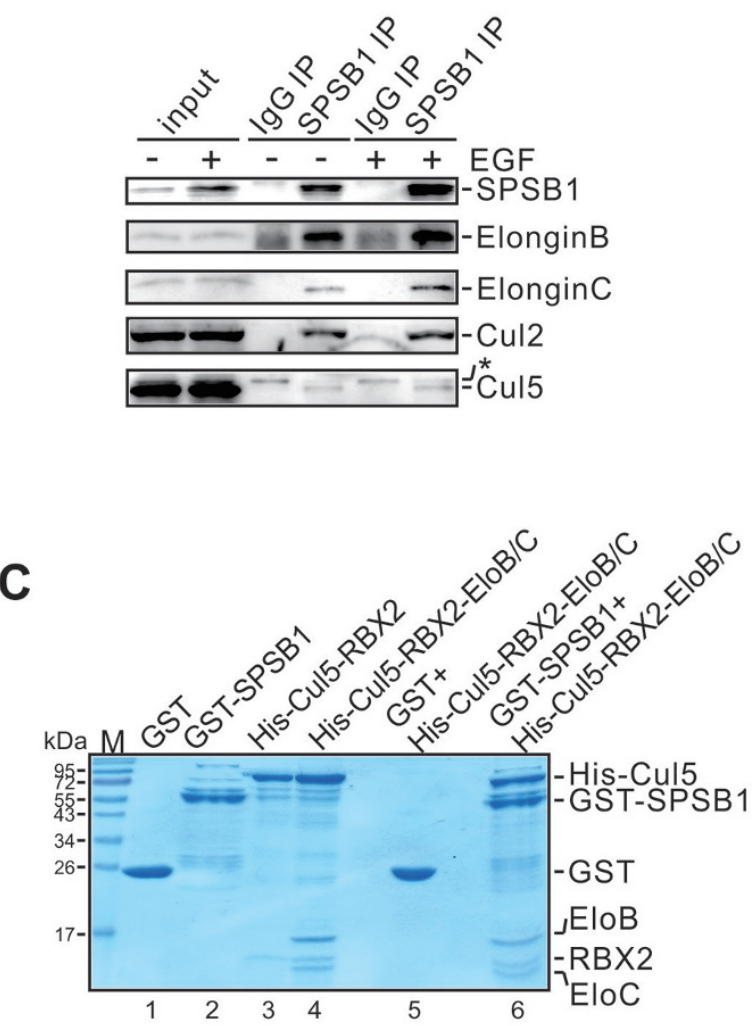

B
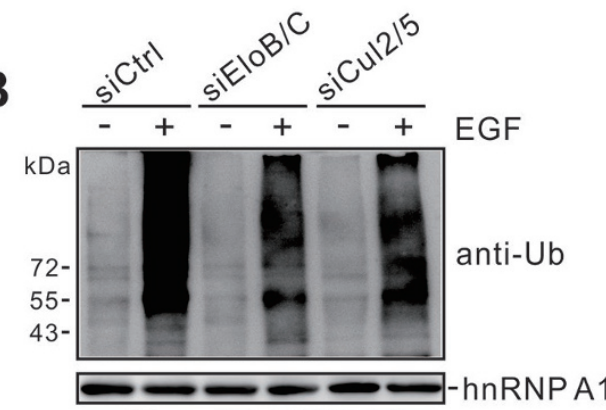

IP: anti-hnRNP A1

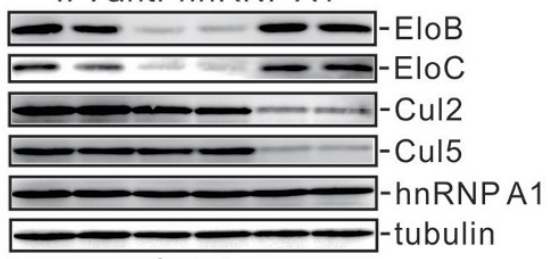

input

D

Figure 3 SPSB1 recruits Elongin B/C-Cullin complexes to ubiquitylate hnRNP A1 both in vivo and in vitro. (A) Endogenous SPSB1 interacts with the components of Elongin B/C-Cul2/5 complexes. Immunoprecipitation was performed with anti-SPSB1 antibody immobilized on Protein A Sepharose beads in the presence of DNase I and RNase A followed by immunoblotting using antibodies against SPSB1, Elongin B, Elongin C, Cul2 or Cul5 (the asterisk represents a non-specific band). (B) Elongin B/C-Cul2/5 complexes mediate EGF-induced hnRNP A1 ubiquitylation. Immunoprecipitated endogenous hnRNP A1 from control knockdown, Elongin B/C double knockdown or Cul2/Cul5 double knockdown HeLa cells treated with or without EGF was immunoblotted with anti-Ub antibody. (C) GST-SPSB1 protein interacts with Elongin B/C-Cul5-RBX2 complex in vitro. GST and GST-SPSB1 immobilized on Glutathione Sepharose were incubated with Elongin B/C and RBX2 co-purified with His-tagged Cul5. After washing with buffer containing $300 \mathrm{mM} \mathrm{KCl}$, bound protein was separated by SDS-PAGE and visualized by Coomassie blue staining. (D) GST-SPSB1 recruits Elongin B/C-Cul5-RBX2 complex to ubiquitylate GST-hnRNP A1 in vitro. An in vitro ubiquitylation assay was carried out using the recombinant GST-hnRNP A1, His-tagged Ub, UBA1 as $\mathrm{E} 1$, UbcH5a as E2, and as well as GST-SPSB1 and purified Elongin B/C-Cul5-RBX2 complex as E3 in the combination as indicated followed by immunoblotting with anti-hnRNP A1 antibody. 
A

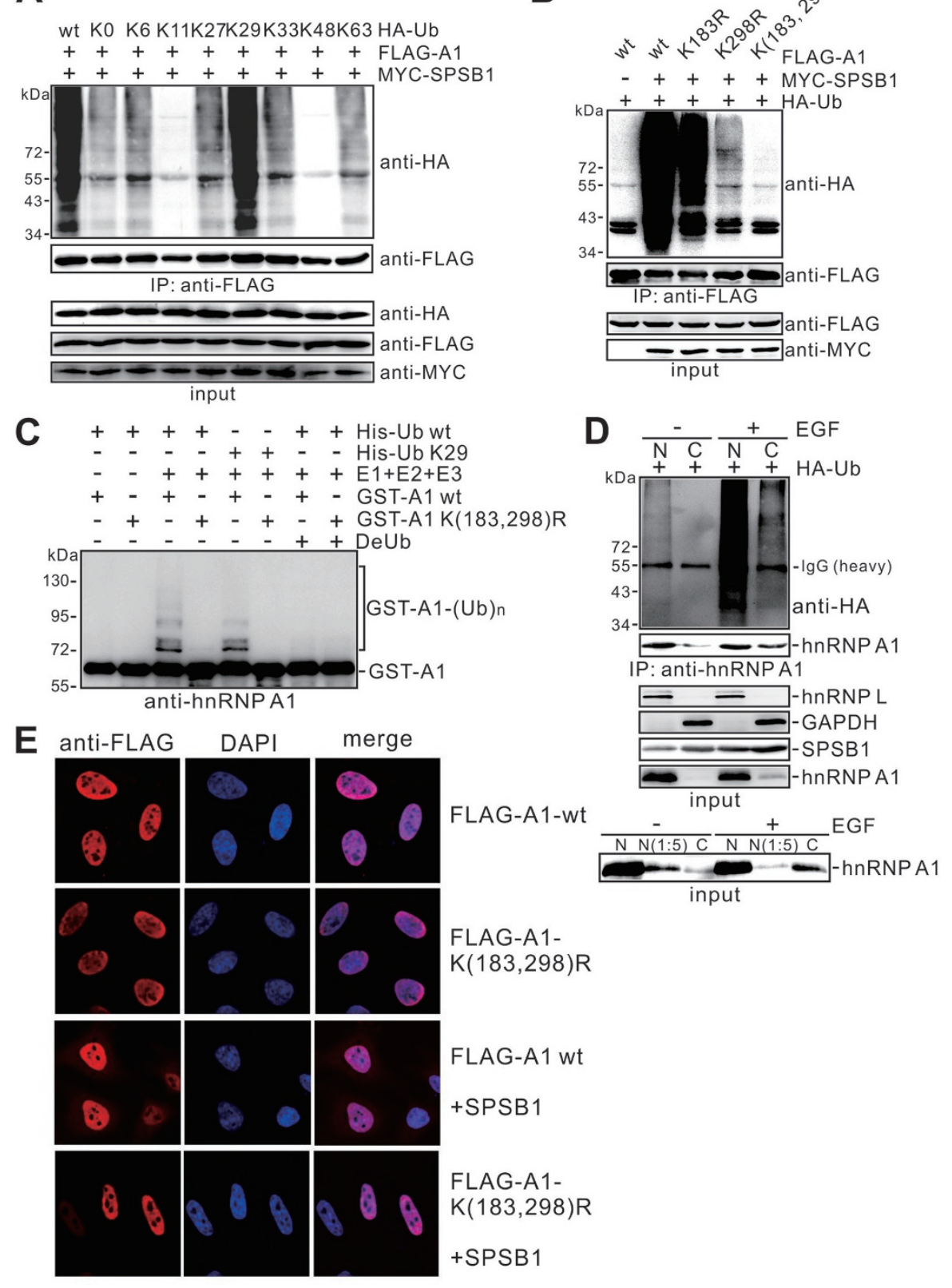

Figure 4 SPSB1 mediates the conjugation of K29-linked polyUb chains onto hnRNP A1 mainly in the nucleus. (A) SPSB1 induces K29-linkage type polyUb on hnRNP A1. Wild-type and mutant Ubs were co-transfected with FLAG-tagged hnRNP A1 and MYC-tagged SPSB1 into HEK 293 cells. Immunoprecipitation was performed with anti-FLAG M2 beads followed by immunoblotting using anti-HA antibody. (B) Mapping the ubiquitylation sites on hnRNP A1. Immunoprecipitation and immunoblotting were carried out as in A. (C) Elongin B/C-Cul5-SPSB1 complex conjugates K29-linked Ub chains on Lys183 and Lys293 of recombinant GST-hnRNP A1 in vitro. The in vitro ubiquitylation assay was performed as in Figure 3D. Usp2cc was used as a deubiquitinating enzyme. (D) EGF-induced hnRNP A1 ubiquitylation takes place mainly in the nucleus and leads to retention of a fraction of hnRNP A1 in the cytoplasm. HeLa cells expressing HA-tagged Ub were subject to nuclear and cytoplasmic separation after control or EGF treatment. Endogenous hnRNP A1 immunoprecipitated by anti-hnRNP A1 antibody was immunoblotted by anti-HA or anti-hnRNP A1 antibody. HnRNP L and GAPDH served as markers for nuclear and cytoplasmic fractions, respectively. The amounts of hnRNP A1 and SPSB1 in nucleus and cytoplasm were assessed by immunoblotting. A 1:5 dilution of the nuclear fractions extracted from cells with or without EGF treatment was made to compare the expression levels of hnRNP A1 in the nucleus and cytoplasm (lower panel). (E) SPSB1-mediated ubiquitylation of hnRNP A1 induces the cytoplasmic localization of a fraction of hnRNP A1. FLAG-tagged wild-type hnRNP A1 and its K(183, 298)R mutant were co-expressed with SPSB1 and immunostained with anti-FLAG antibody. 
uitylation to a similar significant degree (Supplementary information, Figure S3B and S3C). Only mutation of Lys 183 among the three lysines (Lys166, Lys179 and Lys183) between amino acid residues 161 and 185 resulted in a decreased level of hnRNP A1 ubiquitylation (Supplementary information, Figure S3D). In addition, mutation at Lys 298 but not Lys277 at the C-terminus of hnRNP A1 also reduced hnRNP A1 ubiquitylation dramatically (Supplementary information, Figure S3B and S3D). Taken together, Lys183 and Lys298 are the two major sites for SPSB1-mediated ubiquitylation of hnRNP A1 (Figure 4B and Supplementary information, S3E). Lysine-to-arginine substitutions at Lys 183 and Lys298 in hnRNP A1 (K(183, 298)R), and of Lys29 in Ub (K29R) dramatically abolished hnRNP A1 ubiquitylation (Figure 4B and Supplementary information, Figure S3E). Results from in vitro ubiquitylation reconstitution assay demonstrated that both the wild-type $\mathrm{Ub}$ and mutant $\mathrm{Ub}(\mathrm{Ub}$ K29) that carries a single lysine at residue 29 in Ub could conjugate polyUb chains onto the wild-type hnRNP A1 but not the double mutant hnRNP A1 $(\mathrm{K}(183,298) \mathrm{R}$, Figure 4C). The Ub signal on hnRNP A1 was eliminated after incubation with a deubiquitinating enzyme, Usp2cc. In addition, these two lysine sites of hnRNP A1 were identified as predominant $\mathrm{Ub}$ sites on tryptic peptides of immuno-purified hnRNP A1 by LC-MS/MS analysis (Supplementary information, Figure S4A and S4B). A K29-E-GG peptide of Ub was only detected with purified wild-type hnRNP A1 from cells overexpressing SPSB1, but not with the mutant hnRNP A1 $(K(183,298) R$, Supplementary information, Figure S4C). We conclude that SPSB1 mediates the attachment of K29-linked polyUb chains onto K183 and K298 residues of hnRNP A1.

To investigate the effect of hnRNP A1 ubiquitylation on its cellular localization, we performed cytoplasmic and nuclear fractionation. An elevated level of SPSB1 in both cytoplasmic and nuclear fractions was detected in HeLa cells after EGF treatment (Figure 4D). Similar result was shown by immunostaining using anti-SPSB1 antibody (Supplementary information, Figure S5). Ubiquitylated hnRNP A1 was mainly localized in the nucleus after EGF treatment (Figure 4D). However, compared with cells with control treatment, EGF treatment led to the retention of $\sim 20 \%$ of hnRNP A1 in the cytoplasm and simultaneous reduction of the nuclear hnRNP A1 (Figure 4D). Ectopically expressed SPSB1 induced cytoplasmic localization of a fraction of the wild-type hnRNP A1, but not the $\mathrm{K}(183,298) \mathrm{R}$ mutant hnRNP A1 (Figure 4E). Together, our data indicate that EGF-stimulated ubiquitylation of hnRNP A1 takes place mainly in the nucleus and induces a proportion of hnRNP A1 to be sequestered in the cytoplasm.
SPSB1 and ubiquitylation of hnRNP Al have a critical role in mediating $E G F$-stimulated cell migration

$\mathrm{EGF} / \mathrm{EGFR}$ signaling is crucial for promoting cell migration and tumor metastasis. Thus, we next asked whether EGF signaling-induced ubiquitylation of hnRNP A1 has a role in regulating cell migration. In a transwell migration assay, ectopically expressed SPBS1 alone significantly stimulated cell motility (Figure 5A, 5D and $5 \mathrm{G})$, whereas knockdown of endogenous SPSB1 significantly inhibited the activating effect of EGF on cell motility (Figure 5B, 5E and $5 \mathrm{H}$ ). To further test the role of SPSB1-mediated hnRNP A1 ubiquitylation in cell migration directly, we established two cells lines expressing wild-type hnRNP A1 or K(183, 298)R mutant, after depletion of the endogenous hnRNP A1. The mutant hnRNP A1 K(183, 298)R-expressing cells exhibited a lower level of cell motility upon EGF treatment, compared with cells expressing the wild-type hnRNP A1 (Figure $5 \mathrm{C}, 5 \mathrm{~F}$ and $5 \mathrm{I}$ ), indicating that SPSB1 and ubiquitylation of hnRNP A1 are important for mediating EGF-induced cell migration.

\section{SPSB1-mediated ubiquitylation of hnRNP A1 controls} alternative splicing of Racl upon EGF treatment

Rac1, a member of the Ras superfamily of small GTP-binding proteins, acts as a molecular switch to control cytoskeletal rearrangement and cell migration. A Rac1 splicing isoform, named Rac1b, which carries an additional exon $3 \mathrm{~b}$ encoding 19 amino acid residues (Figure 6A), was upregulated in colon, breast and lung cancers [27-29]. To further pinpoint the mechanism by which SPSB1-mediated hnRNP A1 ubiquitylation regulates cell migration, we first examined the splicing pattern of Racl, a known hnRNP A1 target. HnRNP A1 has been shown to bind to a silencer element in Racl exon $3 \mathrm{~b}$ and inhibit exon $3 \mathrm{~b}$ splicing, therefore promoting the generation of Rac1 lacking exon $3 \mathrm{~b}$ whereas suppressing the production of Raclb [30]. We found that the splicing of exon $3 b$ was activated upon EGF treatment in HeLa cells (Figure 6B, upper panel). Results from immunoblotting analysis also revealed that EGF enhanced Rac1b expression but had no effect on total Rac1 expression in HeLa, A549 and EpH4 cells (Figure 6B, lower panel; Supplementary information, Figure S2C and S2D). However, knockdown of SPSB1 could almost completely abolish EGF-induced upregulation of Raclb without affecting the expression level of total Rac1 (Figure 6B), indicating that SPSB1 is required for EGF-stimulated generation of Rac1b isoform.

In both cell lines we used previously (in Figure 5C, $5 \mathrm{~F}$ and $5 \mathrm{I}$ ), mutations at the two ubiquitylation sites in hnRNP A1 $(\mathrm{K}(183,298) \mathrm{R})$ impaired EGF-stimulated 
A

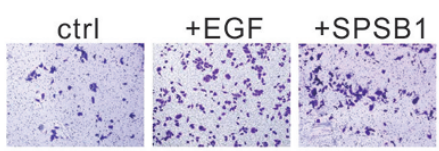

D

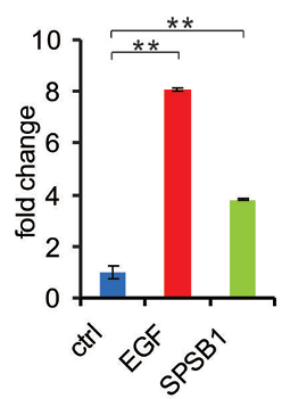

G

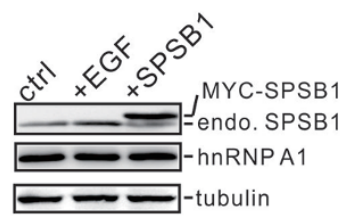

B

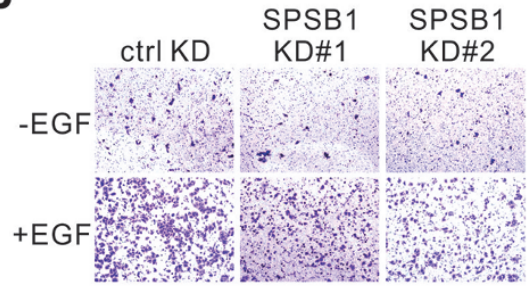

E

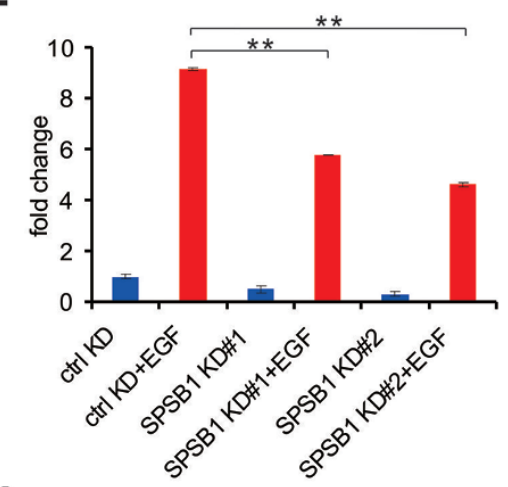

H

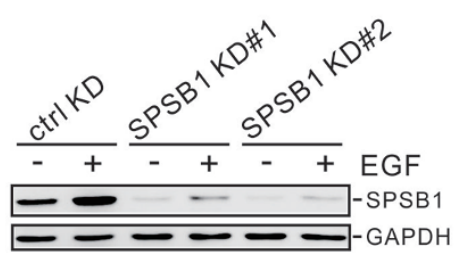

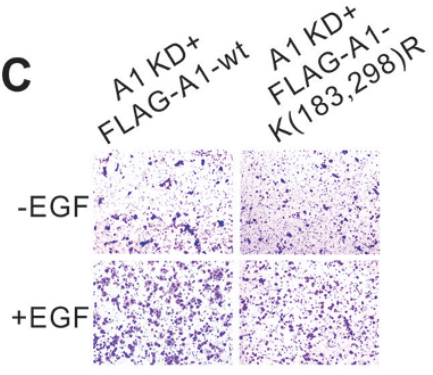

$\mathbf{F}$

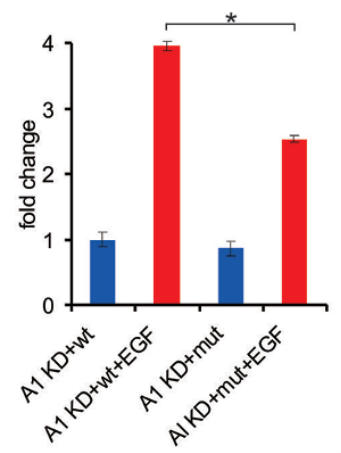

I

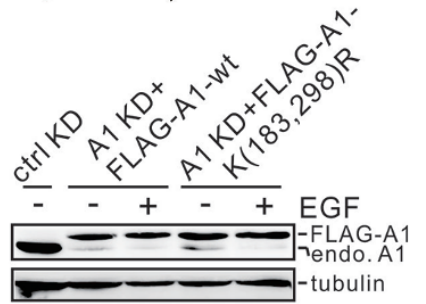

Figure 5 SPSB1-induced hnRNP A1 ubiquitylation mediates EGF-stimulated cell migration. (A, D, G) EGF treatment and ectopic expression of SPSB1 promote cell migration. (B, E, H) Knockdown of SPSB1 impairs EGF-induced cell migration. (C, F, I) HnRNP A1 ubiquitylation mediates EGF-induced cell migration. (A, B, C) Transwell assays were performed with HeLa cells under the indicated treatments. (D, E, F) Quantitation of results in A, B and $\mathbf{C}\left({ }^{*} p<0.05,{ }^{* *} p<0.01\right.$, Student's $t$-test). Error bars represent s.d. $(n=5)$. (G, H, I) Immunoblotting analyses of the expression levels of SPSB1 and hnRNP A1 in HeLa cells subjected to the indicated treatments.

exon $3 \mathrm{~b}$ inclusion and Rac1b expression (Figure 6C), suggesting that ubiquitylation of hnRNP A1 mediates EGF-induced Racl exon $3 \mathrm{~b}$ inclusion. To test whether the ubiquitylation-deficient mutant hnRNP A1 $(\mathrm{K}(183,298)$ $\mathrm{R})$ acts in a dominant negative manner in the de-repression of Racl splicing, wild type or mutant hnRNP A1 was ectopically expressed in HeLa cells with or without EGF treatment. Both wild-type and mutant hnRNP A1 inhibited exon $3 \mathrm{~b}$ inclusion in the absence of EGF. However, hnRNP A1 mutant $(\mathrm{K}(183,298) \mathrm{R})$ had much stronger effect on blocking exon $3 \mathrm{~b}$ splicing than wild-type hnRNP A1 in the presence of EGF (Figure 6D). These results indicate that EGF-induced hnRNP A1 ubiquitylation leads to inactivation of hnRNP A1, preventing it from repressing $R a c 1$ exon $3 \mathrm{~b}$ splicing.

We noticed that in the rescue experiment, hnRNP A1 mutant $(\mathrm{K}(183,298) \mathrm{R})$ only partially blocked EGF-induced exon $3 \mathrm{~b}$ inclusion (Figure $6 \mathrm{C}$ ), suggesting that other factors, in addition to hnRNP A1, might participate into EGF-induced Racl splicing. This possibility was further confirmed by knockdown of hnRNP A1 in HeLa cells with or without EGF treatment (Figure 6E). Although, upon EGF-treatment, the inclusion ratio of exon $3 b$ in hnRNP A1-knockdown cells was comparable to that in control cells, splicing of exon $3 \mathrm{~b}$ in hnRNP A1-knockdown cells in the absence of EGF was stimulated to a less extent compared with the control cells after EGF treatment (Figure 6E, lanes 2-5). The SRPK family of kinases are capable of phosphorylating the RS domain of SR proteins and participating in both constitutive and alternative splicing via controlling the subcellular localization and splicing activity of SR proteins. It was 
reported previously that phosphorylation of SR protein by SRPK can regulate Racl splicing in colon cancer cells [31], and that Akt activation enhances SRPK nuclear translocation and SR protein phosphorylation to control EGF-induced changes in splicing [11]. Moreover, in the work from Zhou et al., Racl exon 3b splicing was identified as an EGF-activated splicing event, which partially responded to Akt inhibitor Wortmannin (see Supplementary information, Table S1 in Ref [11]). These results strongly suggest that the SR-SRPK pathway contributes to EGF-induced Racl splicing. Indeed, when both SRPK1 and SRPK2 were depleted, Rac1 splicing also responded partially to EGF treatment (Figure 6E, lanes 6 and 7). Interestingly, when hnRNP A1, SRPK1 and SRPK2 were depleted simultaneously, Racl splicing became insensitive to EGF treatment, and consequently, the inclusion ratio of exon $3 \mathrm{~b}$ was comparable to that in hnRNP A1 knockdown cells in absence of EGF treatment (Figure 6E, lanes 4, 8 and 9). Moreover, the protein level of Rac $1 b$ isoform was closely correlated with the mRNA level under these conditions, whereas the total Rac1 protein level remained almost unchanged. Altogether, these data clearly indicate that both hnRNP A1 and SRPK1/2 contribute to EGF-stimulated Rac1 splicing.
A

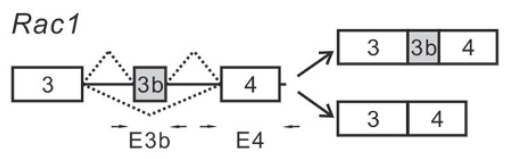

B

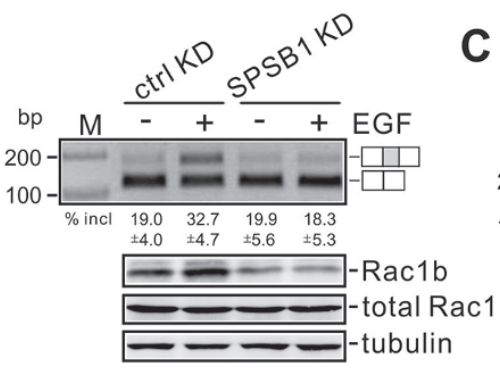

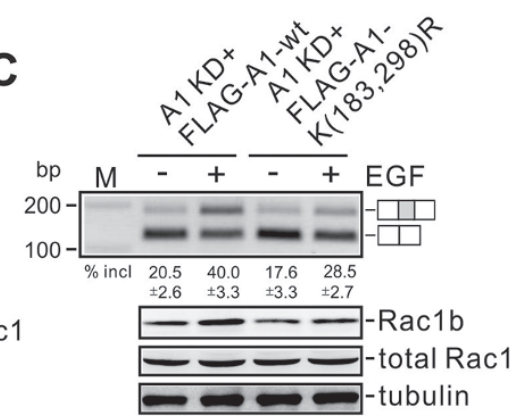

E
D

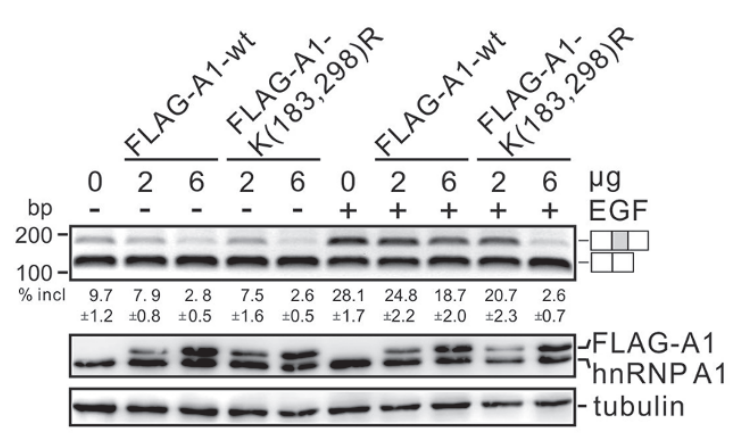

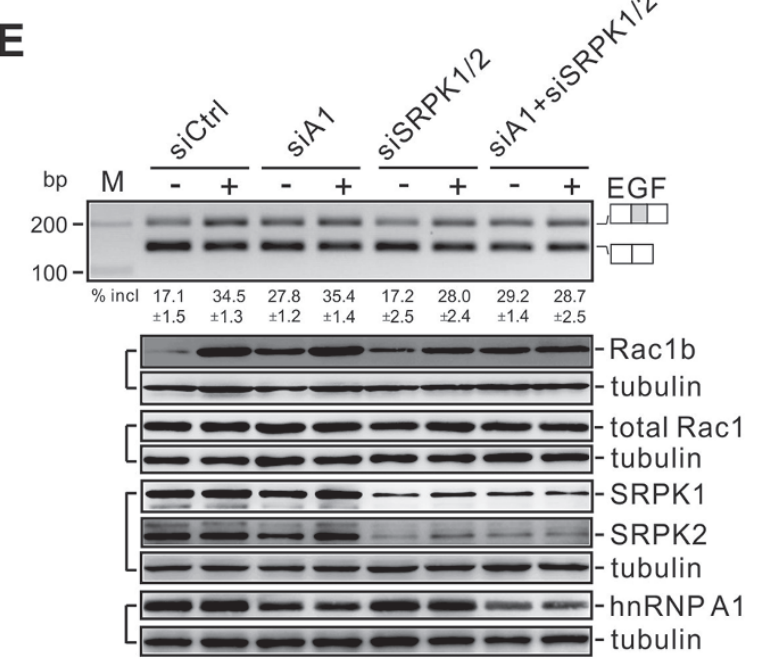

Figure 6 SPSB1-mediated ubiquitylation of hnRNP A1 activates Rac1 isoform, Rac1b, in response to EGF. (A) Schematic representation of Rac1 pre-mRNA. Exons are represented by boxes, introns by lines and splicing pattern by dotted lines. (B) RT-PCR and western blot analyses of Rac1 splicing patterns in control knockdown or SPSB1-knockdown cells treated with or without EGF. The average percentages of exon inclusion with s.d. are shown below $(n=3)$. (C) RT-PCR and Western blot analyses of Rac1 splicing patterns in control knockdown, hnRNP A1 knockdown or hnRNP A1 knockdown cells rescued by the wild-type or mutant hnRNP A1 under control or EGF treatment. The average percentages of exon inclusion with s.d. are shown below $(n=3)$. (D) RT-PCR analysis of Rac1 splicing patterns in cells transfected with different amounts of wild-type or mutant hnRNP A1 expression plasmids under control or EGF treatment. The average percentages of exon inclusion with s.d. are shown below $(n=3)$. Brackets indicate that the immunoblotting detection was done on the same gel by cutting or stripping the membrane. (E) RT-PCR analysis of Rac1 splicing patterns in control, hnRNP A1, SRPK1/SRPK2 double, or hnRNP A1/SRPK1/SRPK2 triple knockdown cells with or without EGF-treatment. The average percentages of exon inclusion with s.d. are shown below $(n=3)$. Western blot signals within the brackets on the left were obtained from the same gel. 
A

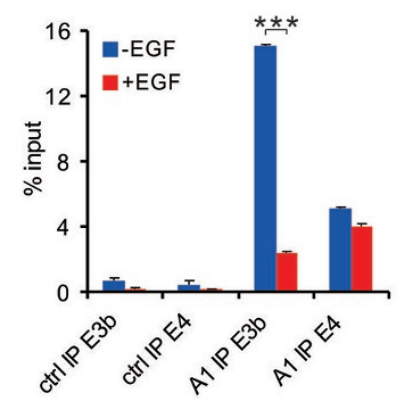

C

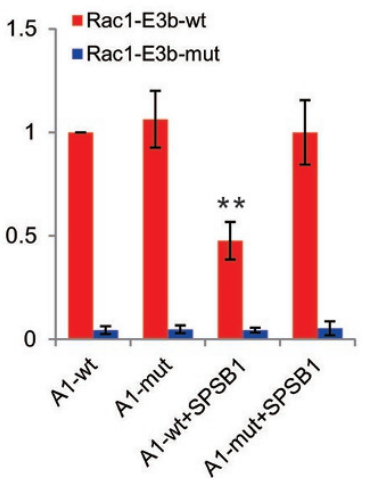

B
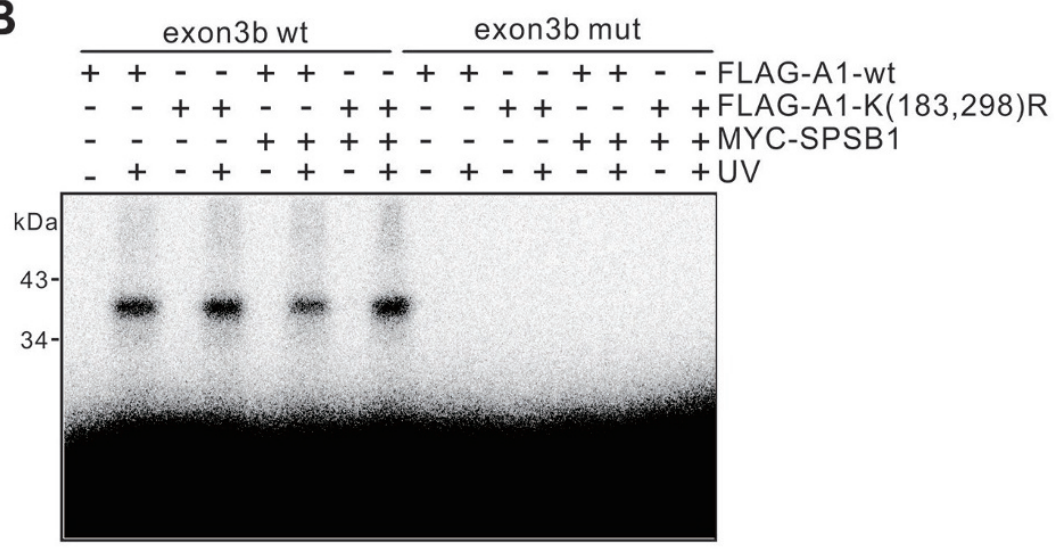

D

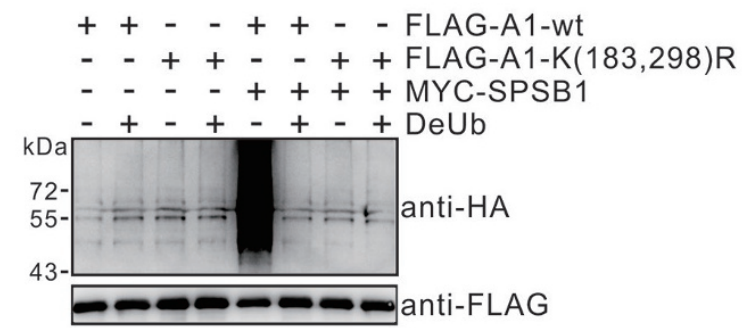

Figure 7 Ubiquitylated hnRNP A1 has a reduced RNA-binding activity, which may result in its release from Rac1 pre-mRNA upon EGF treatment. (A) RIP assay shows that EGF-induced ubiquitylation of hnRNP A1 leads to its release from exon $3 \mathrm{~b}$ of Rac1. RNA-protein complex was immunoprecipitated by control or anti-hnRNP A1 antibody and isolated RNA was detected by RT-PCR using primers indicated in $\mathbf{A}\left({ }^{* * *} p<0.001\right.$, Student's $t$-test). (B) Ubiquitylation of hnRNP A1 leads to a decrease in its RNA binding activity to its target pre-mRNA. ${ }^{32}$ P-labeled RNA transcripts containing wild-type and mutant Rac1 exon $3 b$ sequences were UV-crosslinked to FLAG-tagged wild-type and mutant hnRNP A1 purified from cells with or without SPSB1 overexpression followed by RNase A digestion and SDS-PAGE. (C) Quantitation of three independent UV crosslinking assay results for $\mathbf{B}\left({ }^{* *} p<0.01\right.$, Student's $t$-test). (D) Normalization of FLAG-tagged wild-type and mutant hnRNP A1 for UV crosslinking assay in $\mathbf{C}$ by incubation them with deubiquitinating enzyme Usp2cc.

We also identified several other EGF-induced splicing events such as FAM13B, MBNL1 and RBM10 (Supplementary information, Table S3), which seemed to be primarily regulated by hnRNP A1 (Supplementary information, Figure S6). Upon hnRNP A1 knockdown, the splicing patterns of $F A M 13 B, M B N L 1$ and $R B M 10$ were similar to those in EGF-treated control cells and became insensitive to EGF treatment. Furthermore, expression of ubiquitylation-deficient hnRNP A1 in hnRNP A1 knockdown cells failed to induce splicing changes of FAM13B, $M B N L 1$ and RBM10 under EGF treatment (Supplementary information, Figure S6). Therefore, in contrast to Rac1 splicing, the ubiquitylation of hnRNP A1 has a dominant role in regulating the splicing of FAM13B, MBNL1 and RBM10 upon EGF stimulation.
EGF-induced hnRNP Al ubiquitylation results in its release from Racl pre- $m R N A$, likely due to its reduced RNA binding activity

To mechanistically understand the impact of hnRNP A1 ubiquitylation on splicing regulation, we examined binding of endogenous hnRNP A1 to Racl pre-mRNAs by RNA immunoprecipitation (RIP) coupled with UV crosslinking. Notably, endogenous hnRNP A1 bound specifically to exon $3 \mathrm{~b}$, but the binding affinity decreased dramatically upon EGF treatment (Figure 7A). As a control, the binding of hnRNP A1 to exon 4 of Racl was not affected by EGF (Figure 7A). These data indicate that EGF treatment leads to the release of hnRNP A1 from exon $3 b$, resulting in the de-repression of exon $3 b$ inclusion. This could be due to a reduced level of hnRNP A1 
in the nucleus (Figure 4D) and a decreased RNA binding activity of ubiquitylated hnRNP A1. To further test whether ubiquitylation of hnRNP A1 affects its binding to target RNAs, we performed UV crosslinking assay using in vitro transcribed exon $3 \mathrm{~b}$ RNAs carrying the previously identified hnRNP A1-binding site [31]. Wildtype and mutant hnRNP A purified from HEK 293 cells bound specifically to wild-type exon $3 \mathrm{~b}$ RNA with equal affinity, but much less so to exon 3b RNA containing a mutated hnRNP A1-binding site (Figure 7B and 7C). Intriguingly, FLAG-tagged wild-type hnRNP A1 purified from cells ectopically expressing SPSB1 exhibited a lower affinity to wild-type exon 3b RNA as compared with purified hnRNP A1 with mutated Ub sites (Figure 7B and $7 \mathrm{C}$ ). The amounts of purified wild-type and mutant hnRNP A1 used for UV crosslinking assay were normalized after deubiquitylation treatment by Usp2cc (Figure 7D). These data strongly suggest that EGF-induced ubiquitylation of hnRNP A1 affects alternative splicing due to its reduced binding to its pre-mRNA targets in the nucleus.

Stimulation of Racl splicing by ubiquitylation of hnRNP $A 1$ and activation of SRPKs promotes EGF-induced cell migration

We next asked whether the ubiquitylation of hnRNP A1 and the activation of SRPK participate in EGF-promoted cell migration via Racl splicing. We performed transwell assays on control knockdown, hnRNP A1 knockdown, SRPK1/2 knockdown or combined hnRNP A1/SRPK1/2 knockdown cells with or without EGF treatment. In agreement with the results shown in Figure $5 \mathrm{C}, 5 \mathrm{~F}$ and 5I, which suggest a positive role of EGF-induced hnRNP A1 ubiquitylation in promoting cell migration, knockdown of hnRNP A1 resulted in an elevated cell motility in the absence of EGF, but did not further increase cell migration after EGF treatment compared with control cells (Figure 8A and 8B). Notably, knockdown of SRPK1/2 also inhibited EGF-stimulated cell migration. Importantly, combined knockdown of hnRNP A1 and SRPK1/2 was more effective in attenuating EGF-induced cell migration compared with single knockdown (Figure $8 \mathrm{~A}$ and $8 \mathrm{~B}$ ). These data strongly suggest that both hnRNP A1 ubiquitylation and SRPK activation critically contribute to EGF-enhanced cell migration. To examine whether the increased level of Raclb expression is important for EGF-induced cell migration, antisense RNA oligonucleotide (ASO) method [32] was applied to modify the splicing pattern of Racl without affecting mRNA and protein expression levels of total Racl (Figure 8C and 8D). As shown in Figure 8E and 8F, Racl-specific ASO significantly impaired EGF-induced cell migra- tion, indicating that EGF-induced expression of Raclb isoform mediates the promotion of cell migration. Taken together, our study report two converging pathways involving SPSB1-mediated ubiquitylation of hnRNP A1 and the activation of SRPK in controlling cell migration via EGF-regulated Racl splicing.

\section{Discussion}

Our data in this study reveal that SPSB1-mediated ubiquitylation of hnRNP A1 has a critical role in regulating EGF-induced alternative splicing and cell migration. As depicted in Figure 8E, hnRNP A1 is mainly localized in the nucleus and usually represses splicing by binding to alternatively spliced exons. Upon EGF stimulation, expression of SPSB1 is upregulated, which promotes the conjugation of K29-linked polyUb chains onto hnRNP A1 through recruitment of Elongin B/C-Cullin E3 $\mathrm{Ub}$ ligase complexes, leading to cytoplasmic retention of a fraction of hnRNP A1. The ubiquitylated hnRNP A1 is mainly localized in the nucleus, but has a reduced RNA-binding activity toward Racl pre-mRNA. Combination of these two effects leads to the de-repression of Racl exon $3 \mathrm{~b}$ splicing. In parallel, EGF also stimulates signaling via the SR-SRPK axis, which joins forces to activate Racl splicing to promote cell migration. Our data therefore identify a new mechanism underlying EGF signaling-mediated cell migration involving regulated Racl splicing by SPSB1-mediated ubiquitylation of hnRNP A1 and activation of SRPKs.

Protein ubiquitylation is a prominent post-translational modification [33, 34]. Initially, ubiquitylation was identified as a signal for selective degradation of proteins. Later, it appears that the nonproteolytic modifications via Ub chain conjugation at different lysine residues serve as Ub codes, which transduce signals that regulate cellular activities [35, 36]. K29-linked polyubiquitylation is an unconventional type of $\mathrm{Ub}$ modification, and its biological functions are not well understood. Only few studies suggested its roles in protein degradation, protein-protein interaction and mRNP remodeling [37-39]. Prior studies have placed RNA-binding splicing regulators as major players in connecting extracellular stimuli to splicing decisions in the nucleus. The majority of work has focused on signal-induced phosphorylation modification of RNA-binding splicing regulators by kinase cascades. Our findings identify hnRNP A1 as a new substrate of cellular ubiquitin system and unravel a previously unknown role of nonproteolytic ubiquitylation of RNA-binding protein in signal-induced splicing regulation. Thus, our study establishes a link between Ub signaling and alternative splicing, and provides novel insights into the complex 
A

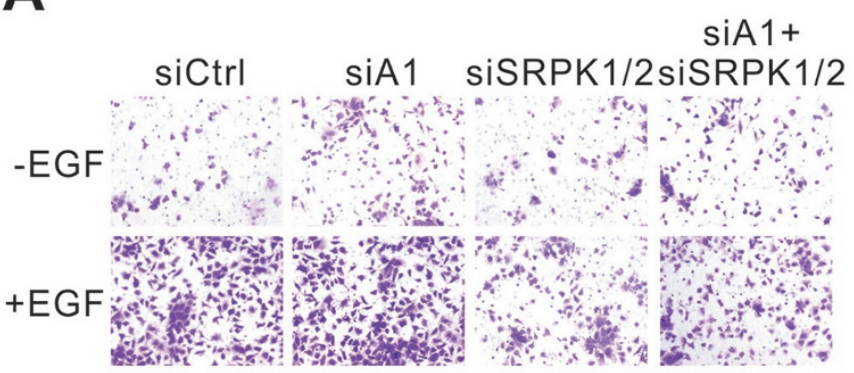

B

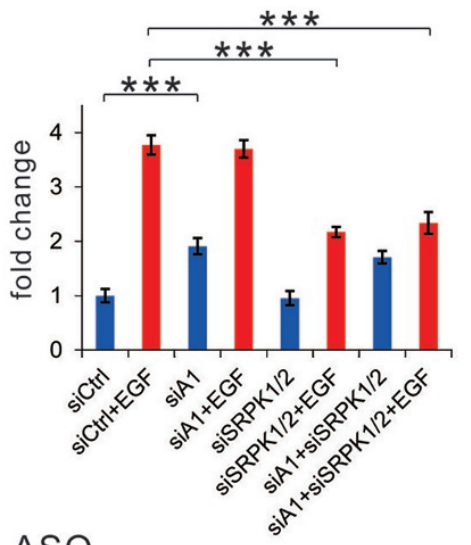

C

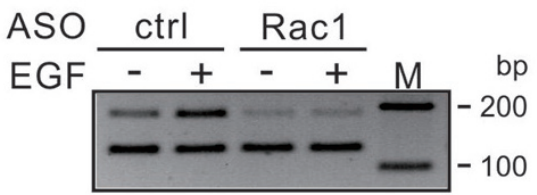

$\mathbf{E}$

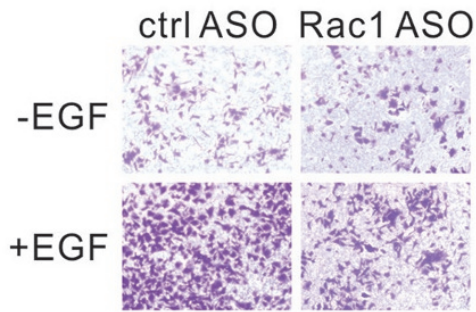

D
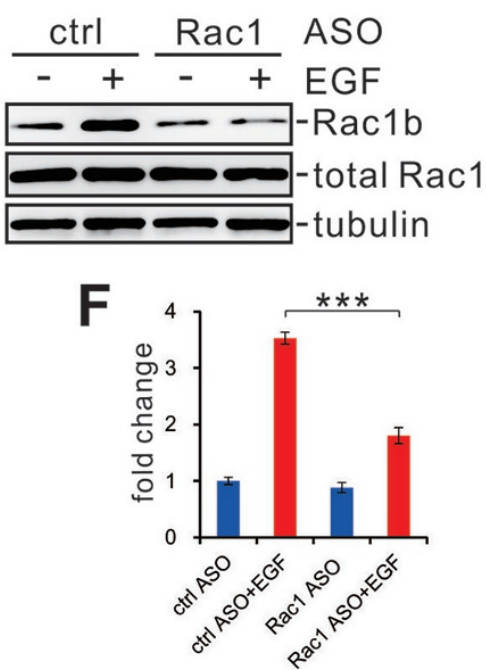

G

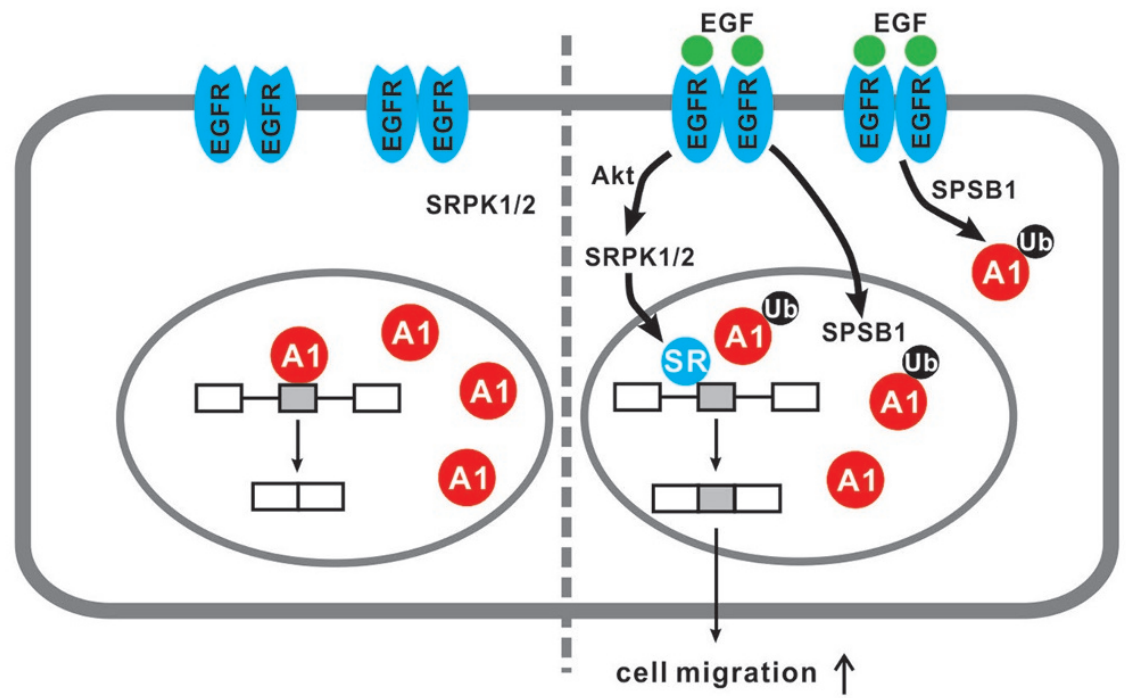

Figure 8 Ubiquitylation of hnRNP A1 together with SRPK1/2 mediates EGF-induced cell migration through regulating Rac1 splicing. (A) Transwell assays were performed with HeLa cells under the indicated treatments. (B) Quantitation of results in $\mathbf{A}\left({ }^{* *} p<0.01,{ }^{* * *} p<0.001\right.$, Student's $t$-test). Error bars represent s.d. $(n=5)$. (C) RT-PCR analysis of Rac1 splicing patterns in cells transfected with control or Rac1-specific ASOs under the control or EGF treatment. (D) Immunoblotting analysis of Rac1b and total Rac1 expression levels in cells with indicated treatment as in C. (E) Splicing-switch ASOs impair EGF-induced cell migration. Transwell assay was performed in cells with indicated treatment as in C. (F) Quantitation of results in $\mathbf{E}$. $\left({ }^{* *} p<0.001\right.$, Student's $t$-test). Error bars represent s.d. $(n=5)$. (G) A working model for EGF-induced cell migration via Rac1 splicing. 
network of signal-induced gene expression regulation involving the unconventional K29-linked polyUb chains.

HnRNP A1 is a member of the core hnRNP particle and known to participate in the regulation of mRNA splicing, stability, export, translation, of telomere maintenance and miRNA biogenesis [40]. HnRNP A1 consists of two N-terminal RNA recognition motifs (RRM1 and RRM2, aa 1-196) and a C-terminal domain carrying several RGG repeats and an M9 motif (aa 268-305). Our data indicate that SPSB1 is required for EGF-induced ubiquitylation of hnRNP Al (Figure 2H), and mediates the attachment of K29-linked polyUb chains to hnRNP A1 preferentially at residues K183 and K298 (Figure 4A$4 \mathrm{C}$ and Supplementary information, Figures S3 and S4). EGF-induced ubiquitylation of hnRNP A1 results in a reduced RNA-binding affinity to its RNA targets (Figure 7). This is very likely due to the $\mathrm{Ub}$ modification at K183, which is localized in the C-terminal end of RRM2. Poly$\mathrm{Ub}$ chains formed on K183 residue may interfere with RNA-protein interaction by changing the conformation of RRM2. It has been previously reported that although hnRNP A1 is predominantly localized in the nucleus, it shuttles rapidly between the nucleus and cytoplasm [41]. Several studies have shown that M9 motif is involved in hnRNP A1 nuclear import and export [42-44]. Ub modification at K298, which is located in M9 motif, may affect hnRNP A1 localization, as a proportion of hnRNP A1 accumulated in the cytoplasm after EGF treatment, and ubiquitylation-deficient hnRNP A1 mutant remained in the nucleus (Figure 4D and 4E). Regulated nuclear import and export of hnRNP A1 can affect not only mRNA splicing but also mRNA transport and translation of hnRNP A1 target genes [45-49]. Thus, besides the role of hnRNP A1 ubiquitylation in regulating pre-mRNA splicing, $\mathrm{Ub}$ modification may modulate the action of hnRNP A1 in other steps of RNA metabolism.

Rac1, a ubiquitously expressed small GTPase, has a crucial role in regulating cell migration by controlling membrane ruffles and lamellipodial protrusion [50]. Through knockdown of Rac1 or expression of a dominant negative mutant of Rac1, previous studies have shown that Rac1 activation is required for EGF-induced cell migration [51-53]. However, the underlying mechanisms remain elusive. Rac1 cycles between an active GTPbound form and an inactive GDP-bound form. The alternatively spliced isoform Rac1b, which carries additional 19 amino acids close to switch II region of Rac1, exhibits reduced intrinsic GTPase activity, increased GDP/ GTP exchange activity and inability to interact with GDP dissociation inhibitor [54, 55]. Upregulation of Rac1b has been shown to result in an increase in cellular reactive oxygen species and to mediate the matrix metallo- proteinase-3 (MMP-3)-induced epithelial-mesenchymal transition [56]. Our results demonstrate that EGF activates the splicing of exon $3 \mathrm{~b}$ in Racl pre-mRNA through SPSB1-mediated ubiquitylation of hnRNP A1, together with SRPK activation, leading to the upregulation of Rac1b (Figure 6). Using isoform switch-specific ASO, we showed that Rac1b is essential for EGF-induced cell migration (Figure 8C and 8D). These data provide a novel mechanism for EGF-induced Rac1 activation and EGF-stimulated cell migration through alternative splicing of Racl driven by SPSB1-mediated hnRNP A1 ubiquitylation and the activation of SRPKs.

Recently, Feng et al. [57] provided the first evidence for a role of SPSB1 in cancer. They identified SPSB1, which is spontaneously upregulated during mammary tumor recurrence, as a key mediator of breast cancer recurrence by potentiating c-MET signaling. Intriguingly, we analyzed the mRNA expression profiling data on matched lung adenocarcinoma pairs from a previous study [58] and found that the expression levels of both SPSB1 and Rac1b, but not that of total Rac1, are upregulated in lung adenocarcinoma (data not shown). Consistently, a recent high-content imaging-based migration screening identified SRPK1 as a breast cancer metastasis determinant, as high expression of SRPK1 strongly correlates with poor disease prognosis and increased metastasis to lung and brain [59]. Taken together, our findings, coupled with altered expression and/or activity of SPSB1, Rac1b and SRPKs in cancers, suggest that targeting SPSB1, Rac1b and SRPKs might provide novel and efficient strategies for treating cancer, particularly in those patients who are resistant to EGFR inhibitors.

Both protein ubiquitylation and alternative pre-mRNA splicing regulate a myriad of cellular processes. Here, we report a novel interplay between these two mechanisms and the pivotal role of this interplay in mediating EGF signal-induced cell migration. Our study, for the first time, reveals a critical role of nonproteolytic ubiquitylation of an RNA-binding protein in a signal transduction pathway. Given that RNA-binding proteins have important roles in post-transcriptional gene expression regulation, the function of $\mathrm{Ub}$ modification of RNA-binding proteins in other physiological and pathological conditions warrants further investigation.

\section{Materials and Methods}

\section{Cell culture, treatment and transfection}

HeLa and HEK 293T cells (ATCC) were maintained in Dulbecco's Modified Eagle's medium supplemented with $10 \%$ fetal bovine serum. For EGF treatment, HeLa cells were first starved for $12 \mathrm{~h}$ followed by adding EGF $(50 \mathrm{ng} / \mathrm{ml})$ for the time indicated in the figures. Plasmids or modified ASOs were transiently trans- 
fected using Lipofectamine 2000 (Invitrogen). To generate recombinant lentivirus, HEK 293 T cells were transfected with lentiviral expression constructs together with two helper plasmids pVSVG and delta-R-8.2 using calcium phosphate method. HeLa cells were infected by recombinant lentiviruses and screened for stable expression of MYC-tagged SPSB1 in the presence of puromycin according to manufacturer's instruction (System Biosciences). Stable knock-down of hnRNP A1 or SPSB1 using retrovirus system was established in HeLa cells according to manufacturer's instruction (Clontech). The target sequences of shRNAs are: hnRNP A1 \#1, 5'-CAGCUGAGGAAGCUCUUCA-3'; hnRNP A1 \#2, 5'-AGCAAGAGAUGGCUAGUGC-3'; SPSB1 \#1, 5'-CGUACCCUGUAUUUAUUCUUU-3'; SPSB1 \#2, 5'- GCUGCAUUCAUGGAACAACAA - $3^{\prime}$.

\section{Oligonucleotides}

The sequences of all the oligonucleotides purchased from Invitrogen, Ribobio (siRNA) or GenePharma (ASO) for this study are listed in Supplementary information, Table S4.

\section{Plasmid construction}

PCR fragments encoding the full-length hnRNP A1 and SPSB1 were amplified from HEK 293 cell cDNA using primer pairs A1-BamHI-F/A1-XhoI-R and SPSB1-HindIII-F/SPSB1-XhoImyc-R, respectively, and inserted into the polylinker region of pcDNA3 (Invitrogen) or pGEX-5X-2 (GE Healthcare) vector. PCR fragments encoding hnRNP A1 or SPSB1 with truncations, deletions or point mutations were made by a two-step PCR using primers listed in the Supplementary information, Table S4. pST39-Rbx2-His-Cullin5 and pST39-ElonginB-CBFbeta-ElonginC-Vif-His were kindly provided by Dr. Xianghui Yu (Jilin University, Changchun, China) [60]. To generate pST39-ElonginB-ElonginC, CBFbeta and Vif-His sequences were removed from pST39-ElonginB-CBFbeta-ElonginC-vif-His. pComp-Rac1exon $3 b$-wt and pComp-Rac1-exon 3b-mut were constructed by insertion of annealed oligonucleotides E3B-wt-for/-rev and E3B-mut-for/-rev separately into EcoRI and HindIII sites of pComp-control [61].

\section{RNA-seq data analysis and motif enrichment analysis}

Total RNAs were processed for single-end $(1 \times 100 \mathrm{nt}) \mathrm{RNA}-$ seq on an Illumina HiSeq2000 platform according to manufacturer's instruction. Data analysis was carried out as previously described [62]. Briefly, we mapped the single-end reads to human reference genome (GRCh38) using TopHat with default parameters. The splicing events were extracted from gene annotations by the standalone version of AStalavista (v3.2) [63]. The "junctions. bed" files produced by TopHat were used to quantify the coverage of inclusion and exclusion junctions for each event. The comparisons of splicing events between samples were made using Fisher's exact tests. To identify cis-elements respond to EGF-induced splicing regulation, five regions (R1-R5) on the significantly changed skipping exons and their flanking introns were scanned for the occurrence of all the 5-mer-nucleotide (Supplementary information, Table S2). To assess the significance of each 5-mer-nucleotide on each region, we randomly selected the same number of exons 1000 times from all the skipping exons and did the same analysis as mentioned above. The $p$-value of each 5-mer-nucleotide in each region was calculated as the frequency of the random selections that had equal or more occurrence than real data.

\section{Western blotting}

Cells were lysed in RIPA buffer containing $50 \mathrm{mM}$ Tris- $\mathrm{Cl} \mathrm{pH}$ 7.4, $150 \mathrm{mM} \mathrm{NaCl}, 1 \%$ NP-40, 1 mM EDTA-free protease inhibitor cocktail (Roche) and $1 \mathrm{mM}$ phenylmethylsulfonyl fluoride (PMSF). Lysates were separated by sodium dodecyl sulfate-polyacrylamide gel electrophoresis (SDS-PAGE) followed by gel transfer to a nitrocellulose membrane (BioRad). The membranes were incubated first with the primary antibodies, and then with secondary antibodies coupled to horseradish peroxidase (HRP). Band signals were detected with an enhanced chemiluminescence system (Thermo Scientific) and visualized by image analyzer (Fujifilm). The primary antibodies used for this study are anti-GAPDH from Kang Cheng Bio-tech, anti- $\gamma$-tubulin and anti-FLAG from Sigma, anti-HA from Roche, anti-MYC, anti-Elongin C, anti-Cul2, anti-Cul5, anti-hnRNP A1 monoclonal antibody and anti-Ub from Santa Cruz, anti-hnRNP A1 polyclonal antibody and anti-Elongin B from Abclonal, anti-pan Akt, anti-p-Akt (S473), anti-Erk1/2 and anti-p- Erk1/2 from CST, anti-Rac1b from Millipore and anti-total Rac1 from Cytoskeleton. The rabbit polyclonal SPSB1 antibody was raised against His-tagged SPSB1 and affinity purified by our lab. The HRP-conjugated secondary antibodies anti-mouse IgG and anti-rabbit IgG were purchased from Promega.

\section{Yeast two-hybrid screen}

The full length of hnRNP A1 ORF was first cloned into donor vector pDONR221, and then transferred into pDEST32 using Gateway Cloning system (Invitrogen). This generated the bait plasmid, pDEST32-hnRNP A1, which contains the in-frame fusion of GAL4 DNA binding domain. The prey vector pDEST22 containing human cDNA collections in-frame fused to the GAL4 activating domain (Invitrogen). Using the empty pDEST22 plasmid as a negative prey control, $\mathrm{Y} 2 \mathrm{H}$ screening was performed by transforming yeast strain (Mav203 strain) that harbors bait vector, pDEST32-hnRNP A1, with the prey vectors carrying human E3 ligase cDNA expression library. Yeast transformants were first grown on the SD-2 agar plate (deficient in Leu, Trp) for selection of yeast cells containing both bait and prey vectors, and then transferred to SD-4 (deficient in Leu, Trp, His and Ura) plates to screen for proteins that potentially interact with hnRNP A1. Colonies grown on the SD-4 plates were picked and streaked onto another SD-4 plates with X-Gal (Sigma). "Positive" colonies were scored for those that not only grew in SD-4 medium but also gave blue color in X-Gal staining assay for $\beta$-galactosidase activity. The prey vectors were recovered from the positive colonies, and sequenced after amplification in Escherichia coli. Typically, each interaction was confirmed by transforming yeast Mav203 cells with the indicated bait and prey vectors, and allowing the transformants to grow on the SD-2 or SD-4 agar plates (with or without X-Gal) for $\sim 3$ days at $30{ }^{\circ} \mathrm{C}$. Images of the colonies on both plates were recorded.

\section{Immunoprecipitation assay}

Cells were lysed in IP buffer (50 mM Tris-Cl, pH 7.5, 150 $\mathrm{mM} \mathrm{KCl}, 0.5 \% \mathrm{NP} 40,1 \mathrm{mM}$ PMSF) at room temperature for 15 min with rotating. The lysate was centrifuged at $13000 \mathrm{rpm}$ for $10 \mathrm{~min}$ at $4{ }^{\circ} \mathrm{C}$. The supernatant was collected and incubated with anti-Flag M2 beads (Sigma) or the appropriate antibodies 
which were previously immobilized on Protein G Sepharose beads (Roche) at $4{ }^{\circ} \mathrm{C}$ for $3 \mathrm{~h}$ with rotating. The beads were subsequently washed three times with IP buffer. For detection of polyUb chains on hnRNP A1, beads were washed with IP buffer supplemented with $0.1 \%$ SDS. The bound material was fractionated by SDSPAGE followed by Western blot analysis.

\section{In vitro ubiquitylation assay}

Recombinant human His-Ub-wt or His-Ub-K29, His-UBA1 (E1), His-UbcH5a (E2), ElonginB/C-His-Cullin5-RBX2, GSTSPSB1, GST-hnRNP A1-wt and GST-hnRNP A1-K(183, 298)R were expressed and purified from Escherichia coli BL21 (DE3) pLysS cells. 500 ng UBA1, 500 ng UbcH5a, $1 \mu$ g co-purified GSTSPSB1 and Elongin B/C-His-Cullin5-RBX2, $1 \mu \mathrm{g}$ GST-hnRNP A1-wt or -K(183, 298)R and $2 \mu \mathrm{g}$ Ub-wt or Ub-K29 were incubated with ubiquitylation buffer containing $25 \mathrm{mM}$ Tris-HCl, $\mathrm{pH}$ 7.6, $5 \mathrm{mM} \mathrm{MgCl}_{2}, 100 \mathrm{mM} \mathrm{NaCl}, 2 \mathrm{mM}$ DTT, $2 \mathrm{mM}$ ATP, $1 \times$ Protease inhibitor cocktail (Roche) at $30{ }^{\circ} \mathrm{C}$ for $1 \mathrm{~h}$. For the deubiquitylation reaction, $1 \mu \mathrm{g}$ purified deubiquitinating enzyme (Usp2cc) was added and incubated for additional $30 \mathrm{~min}$. The reactions were stopped with $5 \times$ SDS loading buffer followed by SDS-PAGE and immunoblotting using anti-hnRNP A1 antibody.

\section{Mass spectrometry analysis of ubiquitylation sites}

FLAG-tagged hnRNP A1 was immuno-purified from cells expressing MYC-tagged SPSB1 and HA-tagged Ub using anti-FLAG M2 beads under denaturing washing condition.

On-beads digestion: beads were firstly washed with $500 \mu 100$ $\mathrm{mM} \mathrm{NH}_{4} \mathrm{HCO}_{3}$ three times and sonicated with $20 \mu 18 \mathrm{M}$ urea in $100 \mathrm{mM}$ Tris-Cl pH 8.5 for $30 \mathrm{~min}$. For optimizing the activity of trypsin, samples were diluted four-fold with $100 \mathrm{mM}$ Tris- $\mathrm{HCl} \mathrm{pH}$ 8.5. Then trypsin (Promega) was added to make a final concentration of $5 \mu \mathrm{g} / \mathrm{ml}$. The samples were then incubated at $37^{\circ} \mathrm{C}$ for $4 \mathrm{~h}$ with shaking at $800 \mathrm{rpm}$. The supernatant was removed into a new tube and stored at $-80^{\circ} \mathrm{C}$. Adding $80 \mu \mathrm{l}$ additional $5 \mu \mathrm{g} / \mathrm{ml}$ trypsin into the sample tube, and then incubated at $37{ }^{\circ} \mathrm{C}$ overnight with shaking at $500 \mathrm{rpm}$. After digestion, the supernatant was removed into a new tube without disturbing beads. Beads were resuspended in $60 \% \mathrm{ACN}(0.1 \%$ Formic Acid) for $5 \mathrm{~min}$, and centrifuged at 1 $000 \mathrm{rpm}$ for $1 \mathrm{~min}$ followed by transferring supernatant into a new tube. Combine three parts of digested supernatant and speedvac to dry out the sample. Before analysis, formic acid was added to a final concentration of $5 \%$.

LC/ tandem MS (MS/MS) analysis of peptide ubiquitylation: the peptide mixture was pressure loaded onto a $15 \mathrm{~cm}$-long pulledtip analytical column $(75 \mu \mathrm{m}$ i.d.) packed with $2.5 \mu \mathrm{m}$ reverse phase beads (Hydro C18, Phenomenex, Torrance, CA) and was washed with buffer A (see the following section for buffer compositions). The column was then placed in-line with an Easy-nLC 1000 nano HPLC (Thermo Scientific, San Jose, CA) for mass spectrometry analysis.

LC condition: the mobile phase and elution gradient used for peptide separation were as follows: $0.1 \%$ formic acid in water as buffer $A$ and $0.1 \%$ formic acid in water/acetonitrile $(20: 80, \mathrm{v} / \mathrm{v})$ as buffer B, 0-5 min, 0\%-2\% B; 5-140 min, 2\%-35\% B; $140-160$ $\min , 35 \%-80 \% \mathrm{~B}$; $160-170 \mathrm{~min}, 80 \% \mathrm{~B} ; 170-171 \mathrm{~min}, 80 \%-0 \% \mathrm{~B}$ and $171-180 \mathrm{~min}, 0 \% \mathrm{~B}$. The flow rate was at $300 \mathrm{nl} / \mathrm{min}$.

Mass spectrometry condition: data-dependent tandem mass spectrometry (MS/MS) analysis was performed with a Q Exactive
Orbitrap mass spectrometer (Thermo Scientific, San Jose, CA). Peptides eluted from the LC column were directly electrosprayed into the mass spectrometer with the application of a distal $1.8-\mathrm{kV}$ spray voltage. A cycle of one full-scan MS spectrum (m/z 300-1 800) was acquired followed by top 19 MS/MS events and a target MS/MS, sequentially generated on the first to the nineteenth most intense ions selected from the full MS spectrum at a $27 \%$ normalized collision energy. The target MS/MS list was added into inclusion list in the MS method during $180 \mathrm{~min}$ data acquiring process. The number of micro scans was one for both MS and MS/ MS scans and the maximum ion injection time was 50 and $100 \mathrm{~ms}$, respectively. The dynamic exclusion settings used were as follows: charge exclusion, 1 and > 8; exclude isotopes, on; and exclusion duration, 30 seconds. MS scan functions and LC solvent gradients were controlled by the Xcalibur data system (Thermo Scientific).

Data analysis: the acquired MS/MS data were analyzed against a UniProtKB Homo sapiens database (database released on 24 September 2015) using Integrated Proteomics Pipeline (IP2, http:// integratedproteomics.com/). To accurately estimate peptide probabilities and false discovery rates, we used a decoy database containing the reversed sequences of all the proteins appended to the target database. To search for ubiquitylation, lysines were treated as differentially modified by +114.0429 Da.

\section{Cell migration assay}

Cell migration was studied using $8-\mathrm{mm}$ transwell chambers with $8-\mu \mathrm{m}$ pores (Corning Costar, Corning). The bottom surface of each membrane was coated with serum-free medium for $1 \mathrm{~h}$. Approximately $10^{5}$ cells were seeded in the upper chambers in 200 $\mu 1$ of serum-free medium. Lower chambers contained $600 \mu 1$ of $20 \%$ serum medium. After the cells were allowed to migrate for 24 $\mathrm{h}$, the medium in the upper chamber was sucked out and cells on the upper side were removed with a cotton swab. Cells on the lower side of the membrane were washed with cold PBS, fixed with methanol and stained with $0.2 \%$ crystal violet in PBS. Cells that migrated through the membrane to the lower surface were counted by light microscopy.

\section{Immunofluorescence microscopy}

Cells were seeded on gelatin-coated cover slips to an appropriate density in $35 \mathrm{~mm}$ plates followed by cell transfection or treatment. After medium removal, cells were fixed with PBS containing 4\% paraformaldehyde for $15 \mathrm{~min}$ and permeabilized with $0.1 \%$ Triton X-100 in PBS. After blocking with PBS containing $2 \%$ bovine serum albumin (BSA) for $30 \mathrm{~min}$, cells were incubated with primary antibody diluted in PBS with $1 \%$ BSA for $3 \mathrm{~h}$. After three washes with PBS, cells were incubated with the appropriate fluorescence-conjugated secondary antibodies for $1 \mathrm{~h}$ at room temperature followed by $10 \mathrm{~min}$ of DAPI (4',6-diamidino-2-phenylindole dihydrochloride) staining for nuclei visualization. Slides were then washed three times and mounted in Mowiol reagent. The stained cells were detected using Olympus BX51 microscope and Leica TCS SP2 laser confocal microscope. The final images were obtained and analyzed by using confocal microscopy with FLUOVIEW viewer software and Adobe Photoshop CS4. Each image is a single $\mathrm{Z}$ section at the same cellular level.

\section{Nuclear and cytoplasmic fractionation}

Cells were trypsinized, collected by centrifugation and washed 
with pre-cold PBS three times. Then cells were resuspended in Hyponotic Buffer (10 mM HEPES, pH 7.9, $1.5 \mathrm{mM} \mathrm{MgCl}_{2}, 10$ $\mathrm{mM} \mathrm{KCl}, 0.5 \% \mathrm{NP}-40$ ) for $10 \mathrm{~min}$ on ice with gentle tapping. Cell lysate was then centrifuged at $3000 \times g$ for $5 \mathrm{~min}$. The suspension (cytoplasm) was collected and centrifuged further at $6000 \times g$ for $10 \mathrm{~min}$ to get rid of nucleus contamination; the pellets (nucleus) were resuspended and washed with Hyponotic Buffer twice.

\section{RNA immunoprecipitation}

Cells were irradiated with UV light at $150 \mathrm{~mJ} / \mathrm{cm}^{2}$ and lyzed in buffer containing $50 \mathrm{mM}$ Tris- $\mathrm{HCl}, \mathrm{pH} 7.4,100 \mathrm{mM} \mathrm{NaCl}, 1 \%$ NP-40, 0.1\% SDS, $0.5 \%$ sodium deoxycholate, protease inhibitor cocktail. RNAs were partially fragmented using RNase A (Qiagen). After centrifugation at $10000 \times g$ for $10 \mathrm{~min}$, an aliquot $(10 \%)$ of supernatant was removed and served as input. The remaining supernatant was immunoprecipitated with either a mouse IgG or a hnRNP A1 antibody immobilized on Protein G Sepharose. The bound RNAs were washed extensively and isolated using TRIzol (Invitrogen, USA) followed by RT-qPCR.

\section{UV crosslinking}

${ }^{32} \mathrm{P}$-labeled RNAs were in vitro transcribed by T7 RNA polymerase (Fermentas) using HindIII-linearized pComp-Rac1-exon 3b-wt or pComp-Rac1-exon 3b-mut as template and incubated with purified hnRNP A1 proteins at $30{ }^{\circ} \mathrm{C}$ for $30 \mathrm{~min}$. The in vitro transcribed RNAs contain two copies of wildtype hnRNP A1 binding sites or mutated sequences: exon $3 \mathrm{~b}$ wt, GGGCGAAUUCGUACGGUAAGGAUAUAA GUACGGUAAGGAUAUAAA; exon3b mut: GGGCGAAUUCUUAUAUCCUUACCGUACUUAUAUCCUUACCGUACA. An aliquot of $1 \mu 1$ of tRNA (5 mg/ $\mathrm{ml}$ ) was added into each reaction to remove non-specific binding of proteins. UV crosslinking was done on ice for 20 min with $254-$ nm UV light. Unprotected RNAs were digested with RNase A (at a final concentration of $1 \mathrm{mg} / \mathrm{ml}$ ) at $37{ }^{\circ} \mathrm{C}$ for $20 \mathrm{~min}$. Crosslinked species were analyzed on a $12.5 \%$ SDS polyacrylamide gel and visualized with a phosphoimager (Fujifilm, Japan).

\section{Accession numbers}

The RNA-Seq data are available at NCBI's GEO database under the accession number GSE92939.

\section{Acknowledgments}

We thank Drs Xiang-Dong Fu, Zhongsheng You and Min Wei for critical reading of the manuscript and invaluable suggestions, and all the members of Jingyi Hui and Ronggui Hu's laboratories, especially Jiancheng Yu, Xiaojuan Yang and Jie Ye for technical assistance. We are grateful to Dr Xianghui Yu for generously providing us expression plasmids for Elongin B/C, Cul5 and RBX1/2, and to Dr Daming Gao for anti-Elongin B/C antibodies. This work was supported by National Natural Science Foundation of China (31570820, 31661143035 and 31370787) to $\mathrm{JH}$ and (31270828, 31470770 and 81525019 ) to RH and by the National Basic Research Program of China (2012CB910800 and 2013CB910900) to RH. RH was also supported by Shanghai Institute of Organic Chemistry, CAS, and the Instrument Developing Project of CAS (YZ201339).

\section{Author Contributions}

FW performed most of the experiments. XF, XF and ZW analyzed RNA-seq data. PC performed Y2H screening. NL, HZ, TTJ and HJ helped with experiments. PW and CCLW performed LS-MS/MS analysis. JH, RH and FW designed the experiments, analyzed data and wrote the manuscript. All authors discussed the results and approved the manuscript.

\section{Competing Financial Interests}

The authors declare no competing financial interests.

\section{References}

1 Chen M, Manley JL. Mechanisms of alternative splicing regulation: insights from molecular and genomics approaches. Nat Rev Mol Cell Biol 2009; 10:741-754.

2 Nilsen TW, Graveley BR. Expansion of the eukaryotic proteome by alternative splicing. Nature 2010; 463:457-463.

3 Fu XD, Ares M Jr. Context-dependent control of alternative splicing by RNA-binding proteins. Nat Rev Genet 2014; 15:689-701.

4 Matera AG, Wang ZF. A day in the life of the spliceosome. Nat Rev Mol Cell Biol 2014; 15:108-121.

5 Lee Y, Rio DC. Mechanisms and regulation of alternative pre-mRNA splicing. Annu Rev Biochem 2015; 84:291-323.

6 Shin C, Manley JL. Cell signalling and the control of pre-mRNA splicing. Nat Rev Mol Cell Biol 2004; 5:727-738.

7 Lynch KW. Regulation of alternative splicing by signal transduction pathways. Adv Exp Med Biol 2007; 623:161-174.

8 Blaustein M, Pelisch F, Srebrow A. Signals, pathways and splicing regulation. Int J Biochem Cell Biol 2007; 39:20312048.

9 Ip JY, Tong A, Pan Q, Topp JD, Blencowe BJ, Lynch KW. Global analysis of alternative splicing during T-cell activation. RNA 2007; 13:563-572.

10 Hartmann B, Castelo R, Blanchette M, Boue S, Rio DC, Valcarcel J. Global analysis of alternative splicing regulation by insulin and wingless signaling in Drosophila cells. Genome Biol 2009; 10:R11.

11 Zhou Z, Qiu J, Liu W, et al. The Akt-SRPK-SR axis constitutes a major pathway in transducing EGF signaling to regulate alternative splicing in the nucleus. Mol Cell 2012; 47:422-433.

12 Kostler WJ, Zeisel A, Korner C, et al. Epidermal growth-factor-induced transcript isoform variation drives mammary cell migration. PloS One 2013; 8:e80566.

13 Martinez NM, Agosto L, Qiu J, et al. Widespread JNK-dependent alternative splicing induces a positive feedback loop through CELF2-mediated regulation of MKK7 during T-cell activation. Genes Dev 2015; 29:2054-2066.

14 Citri A, Yarden Y. EGF-ERBB signalling: towards the systems level. Nat Rev Mol Cell Biol 2006; 7:505-516.

15 Zeng F, Harris RC. Epidermal growth factor, from gene organization to bedside. Semin Cell Dev Biol 2014; 28:2-11.

16 Ray D, Kazan H, Cook KB, et al. A compendium of RNA-binding motifs for decoding gene regulation. Nature 2013; 499:172-177.

17 Choi YD, Dreyfuss G. Isolation of the heterogeneous nuclear RNA-ribonucleoprotein complex (hnRNP): a unique supramolecular assembly. Proc Natl Acad Sci USA 1984; 81:7471- 
7475.

18 Zhu J, Mayeda A, Krainer AR. Exon identity established through differential antagonism between exonic splicing silencer-bound hnRNP A1 and enhancer-bound SR proteins. Mol Cell 2001; 8:1351-1361.

19 Rooke N, Markovtsov V, Cagavi E, Black DL. Roles for SR proteins and hnRNP A1 in the regulation of c-src exon N1. Mol Cell Biol 2003; 23:1874-1884.

20 Tavanez JP, Madl T, Kooshapur H, Sattler M, Valcarcel J. hnRNP A1 proofreads $3^{\prime}$ splice site recognition by U2AF. Mol Cell 2012; 45:314-329.

21 Liu Z, Chen P, Gao H, et al. Ubiquitylation of autophagy receptor Optineurin by HACE1 activates selective autophagy for tumor suppression. Cancer Cell 2014; 26:106-120.

22 Hilton DJ, Richardson RT, Alexander WS, et al. Twenty proteins containing a C-terminal SOCS box form five structural classes. Proc Natl Acad Sci USA 1998; 95:114-119.

23 Linossi EM, Nicholson SE. The SOCS boxuAdapting proteins for ubiquitination and proteasomal degradation. IUBMB Life 2012; 64:316-323.

24 Kamura T, Sato S, Haque D, et al. The Elongin BC complex interacts with the conserved SOCS-box motif present in members of the SOCS, ras, WD-40 repeat, and ankyrin repeat families. Genes Dev 1998; 12:3872-3881.

25 Zhang JG, Farley A, Nicholson SE, et al. The conserved SOCS box motif in suppressors of cytokine signaling binds to elongins $\mathrm{B}$ and $\mathrm{C}$ and may couple bound proteins to proteasomal degradation. Proc Natl Acad Sci USA 1999; 96:20712076.

26 Kamura T, Maenaka K, Kotoshiba S, et al. VHL-box and SOCS-box domains determine binding specificity for Cul2Rbx 1 and Cul5-Rbx2 modules of ubiquitin ligases. Genes Dev 2004; 18:3055-3065.

27 Jordan P, Brazao R, Boavida MG, Gespach C, Chastre E. Cloning of a novel human Raclb splice variant with increased expression in colorectal tumors. Oncogene 1999; 18:68356839.

28 Schnelzer A, Prechtel D, Knaus U, et al. Rac1 in human breast cancer: overexpression, mutation analysis, and characterization of a new isoform, Rac1b. Oncogene 2000; 19:30133020 .

29 Zhou C, Licciulli S, Avila JL, et al. The Rac1 splice form Rac $1 b$ promotes K-ras-induced lung tumorigenesis. Oncogene 2013; 32:903-909.

30 Pelisch F, Khauv D, Risso G, et al. Involvement of hnRNP A1 in the matrix metalloprotease-3-dependent regulation of Rac1 pre-mRNA splicing. J Cell Biochem 2012; 113:2319-2329.

31 Goncalves V, Henriques A, Pereira J, et al. Phosphorylation of SRSF1 by SRPK1 regulates alternative splicing of tumor-related Rac1b in colorectal cells. RNA 2014; 20:474-482.

32 Bauman JA, Li SD, Yang A, Huang L, Kole R. Anti-tumor activity of splice-switching oligonucleotides. Nucleic Acids Res 2010; 38:8348-8356.

33 Varshavsky A. The early history of the ubiquitin field. Protein Sci 2006; 15:647-654.

34 Hochstrasser M. Lingering mysteries of ubiquitin-chain assembly. Cell 2006; 124:27-34.

35 Haglund K, Dikic I. Ubiquitylation and cell signaling. EMBO J 2005; 24:3353-3359.
36 Chen ZJ, Sun LJ. Nonproteolytic functions of ubiquitin in cell signaling. Mol Cell 2009; 33:275-286.

37 Chastagner P, Israel A, Brou C. Itch/AIP4 mediates Deltex degradation through the formation of K29-linked polyubiquitin chains. EMBO Rep 2006; 7:1147-1153.

38 Fei C, Li ZF, Li C, et al. Smurf1-mediated Lys29-linked nonproteolytic polyubiquitination of axin negatively regulates Wnt/beta-catenin signaling. Mol Cell Biol 2013; 33:40954105.

39 Zhou HL, Geng CY, Luo GB, Lou H. The p97-UBXD8 complex destabilizes mRNA by promoting release of ubiquitinated HuR from mRNP. Genes Dev 2013; 27:1046-1058.

40 Jean-Philippe J, Paz S, Caputi M. hnRNP A1: the Swiss army knife of gene expression. Int J Mol Sci 2013; 14:1899919024.

41 Pinol-Roma S, Dreyfuss G. Shuttling of pre-mRNA binding proteins between nucleus and cytoplasm. Nature 1992; 355:730-732.

42 Michael WM, Choi M, Dreyfuss G. A nuclear export signal in hnRNP A1: a signal-mediated, temperature-dependent nuclear protein export pathway. Cell 1995; 83:415-422.

43 Siomi H, Dreyfuss G. A nuclear localization domain in the hnRNP A1 protein. J Cell Biol 1995; 129:551-560.

44 Weighardt F, Biamonti G, Riva S. Nucleo-cytoplasmic distribution of human hnRNP proteins: a search for the targeting domains in hnRNP A1. J Cell Sci 1995; 108:545-555.

45 van der Houven van Oordt W, Diaz-Meco MT, Lozano J, Krainer AR, Moscat J, Caceres JF. The MKK(3/6)-p38-signaling cascade alters the subcellular distribution of hnRNP A1 and modulates alternative splicing regulation. $J$ Cell Biol 2000; 149:307-316.

46 Izaurralde E, Jarmolowski A, Beisel C, Mattaj IW, Dreyfuss G, Fischer U. A role for the M9 transport signal of hnRNP A1 in mRNA nuclear export. J Cell Biol 1997; 137:27-35.

47 Iervolino A, Santilli G, Trotta R, et al. hnRNP A1 nucleocytoplasmic shuttling activity is required for normal myelopoiesis and BCR/ABL leukemogenesis. Mol Cell Biol 2002; 22:22552266.

48 Cammas A, Pileur F, Bonnal S, et al. Cytoplasmic relocalization of heterogeneous nuclear ribonucleoprotein A1 controls translation initiation of specific mRNAs. Mol Biol Cell 2007; 18:5048-5059.

49 Roy R, Durie D, Li H, et al. hnRNPA1 couples nuclear export and translation of specific mRNAs downstream of FGF-2/ S6K2 signalling. Nucleic Acids Res 2014; 42:12483-12497.

50 Parri M, Chiarugi P. Rac and Rho GTPases in cancer cell motility control. Cell Commun Signal 2010; 8:23.

51 Ridley AJ, Paterson HF, Johnston CL, Diekmann D, Hall A. The small GTP-binding protein rac regulates growth factor-induced membrane ruffling. Cell 1992; 70:401-410.

52 Yip SC, El-Sibai M, Coniglio SJ, et al. The distinct roles of Ras and Rac in PI 3-kinase-dependent protrusion during EGF-stimulated cell migration. J Cell Sci 2007; 120:31383146.

53 Dise RS, Frey MR, Whitehead RH, Polk DB. Epidermal growth factor stimulates Rac activation through Src and phosphatidylinositol 3-kinase to promote colonic epithelial cell migration. Am J Physiol Gastrointest Liver Physiol 2008; 294:G276-G285. 
54 Matos P, Collard JG, Jordan P. Tumor-related alternatively spliced Rac1b is not regulated by Rho-GDP dissociation inhibitors and exhibits selective downstream signaling. J Cell Biochem 2003; 278:50442-50448.

55 Fiegen D, Haeusler LC, Blumenstein L, et al. Alternative splicing of Rac1 generates Rac1b, a self-activating GTPase. $J$ Biol Chem 2004; 279:4743-4749.

56 Radisky DC, Levy DD, Littlepage LE, et al. Rac1b and reactive oxygen species mediate MMP-3-induced EMT and genomic instability. Nature 2005; 436:123-127.

57 Feng Y, Pan TC, Pant DK, et al. SPSB1 promotes breast cancer recurrence by potentiating c-MET signaling. Cancer Discov 2014; 4:790-803.

58 Selamat SA, Chung BS, Girard L, et al. Genome-scale analysis of DNA methylation in lung adenocarcinoma and integration with mRNA expression. Genome Res 2012; 22:1197-
1211.

59 van Roosmalen W, Le Devedec SE, Golani O, et al. Tumor cell migration screen identifies SRPK1 as breast cancer metastasis determinant. J Clin Invest 2015; 125:1648-1664.

60 Wang X, Wang X, Wang W, et al. Both Rbx1 and Rbx2 exhibit a functional role in the HIV-1 Vif-Cullin5 E3 ligase complex in vitro. Biochem Biophys Res Commun 2015; 461:624629.

61 Hui J, Reither G, Bindereif A. Novel functional role of CA repeats and hnRNP L in RNA stability. RNA 2003; 9:931-936.

62 Zong FY, Fu X, Wei WJ, et al. The RNA-binding protein QKI suppresses cancer-associated aberrant splicing. PLoS Genet 2014; 10:e1004289.

63 Sammeth M, Foissac S, Guigo R. A general definition and nomenclature for alternative splicing events. PLoS Comput Biol 2008; 4:e1000147.

(Supplementary information is linked to the online version of the paper on the Cell Research website.) 